Supporting Information for

\title{
Protein Vesicles Self-Assembled from Functional Globular Proteins with Different Charge and Size
}

Dylan R. Dautel and Julie A. Champion

School of Chemical and Biomolecular Engineering, Georgia Institute of Technology, 950

Atlantic Drive NW, Atlanta, Georgia 30332, United States 


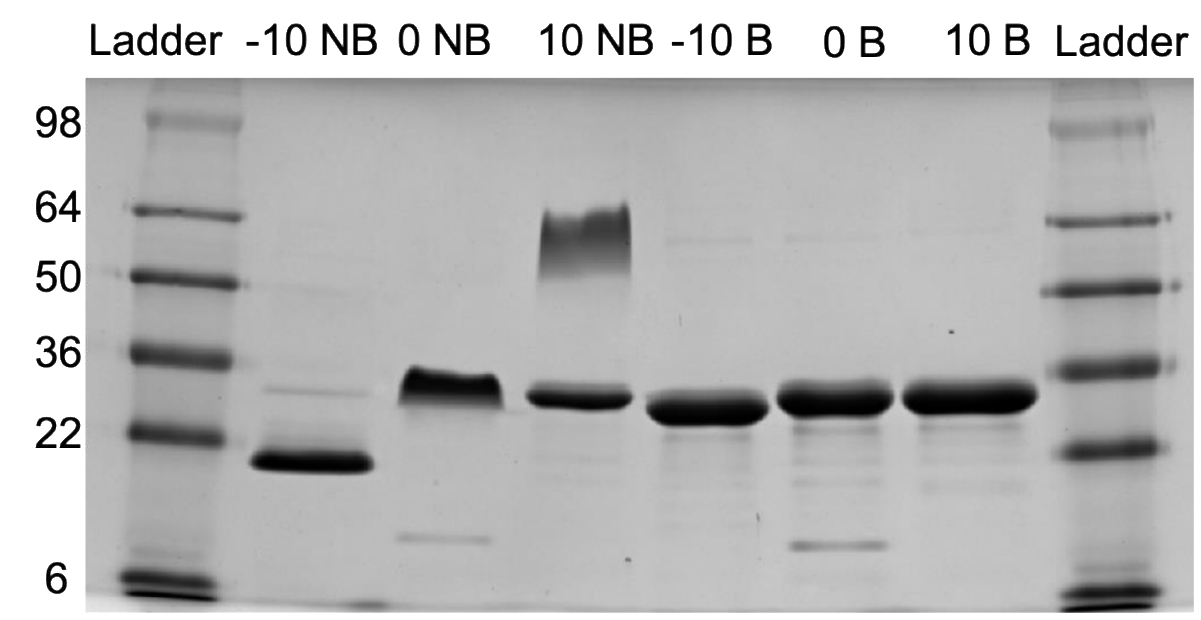

Figure S1. SDS-PAGE gel of sfGFP-ZE. Each charged variant of sfGFP runs differently when not denatured by boiling. The negative variant runs further because there are more negatively charged amino acids on the surface of the protein. The neutral variant runs nearly the same MW as the denatured proteins and the positive variant has two bands one that arrests at a higher MW and another that gets denatured by SDS and runs at the expected MW. The molecular weights of each band on the ladder is shown in $\mathrm{kDa}$. NB- not boiled, B- boiled.

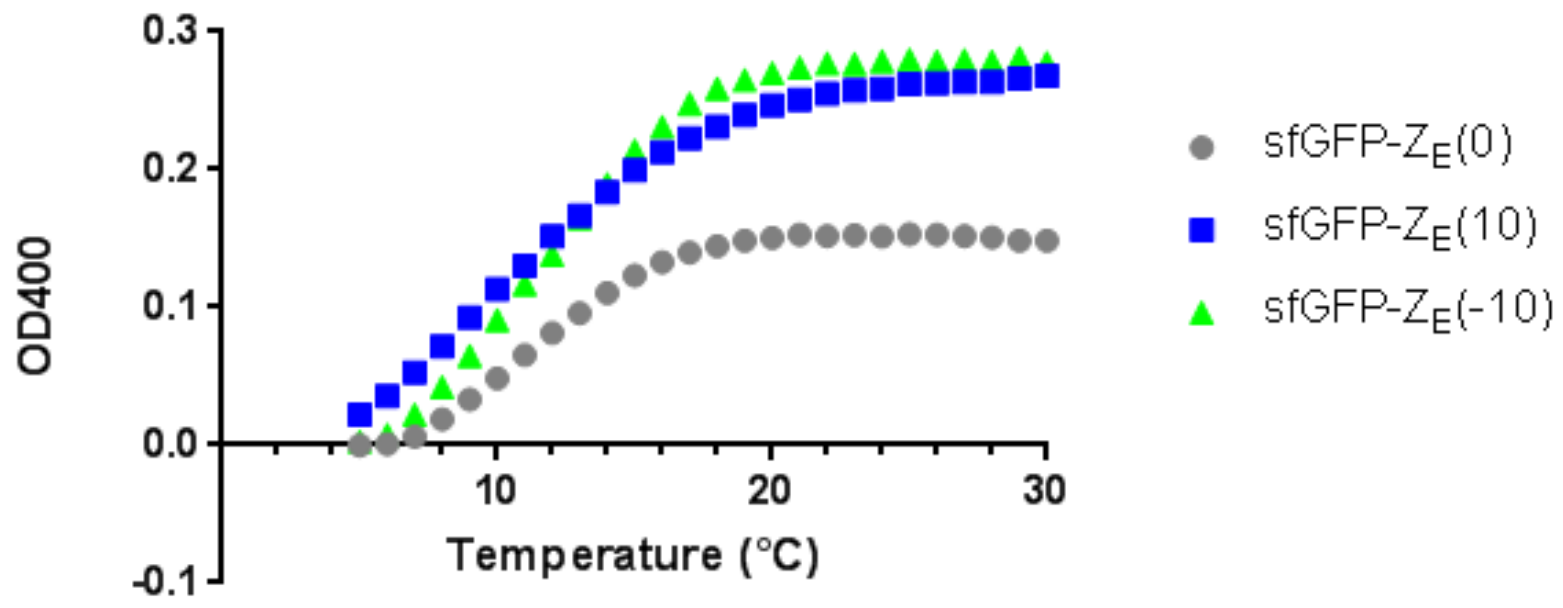

Figure S2. Transition temperature of sfGFP-Z $Z_{\mathrm{E}}$ charge variants. Solutions contain $30 \mu \mathrm{M} \mathrm{Z}_{\mathrm{R}^{-}}$ ELP, $1.5 \mu \mathrm{M}$ sfGFP- $Z_{\mathrm{E}}$, and 1.0 M PBS. The $\mathrm{T}_{\mathrm{t}}$ (point of largest slope) of each variant is $12{ }^{\circ} \mathrm{C}$. 


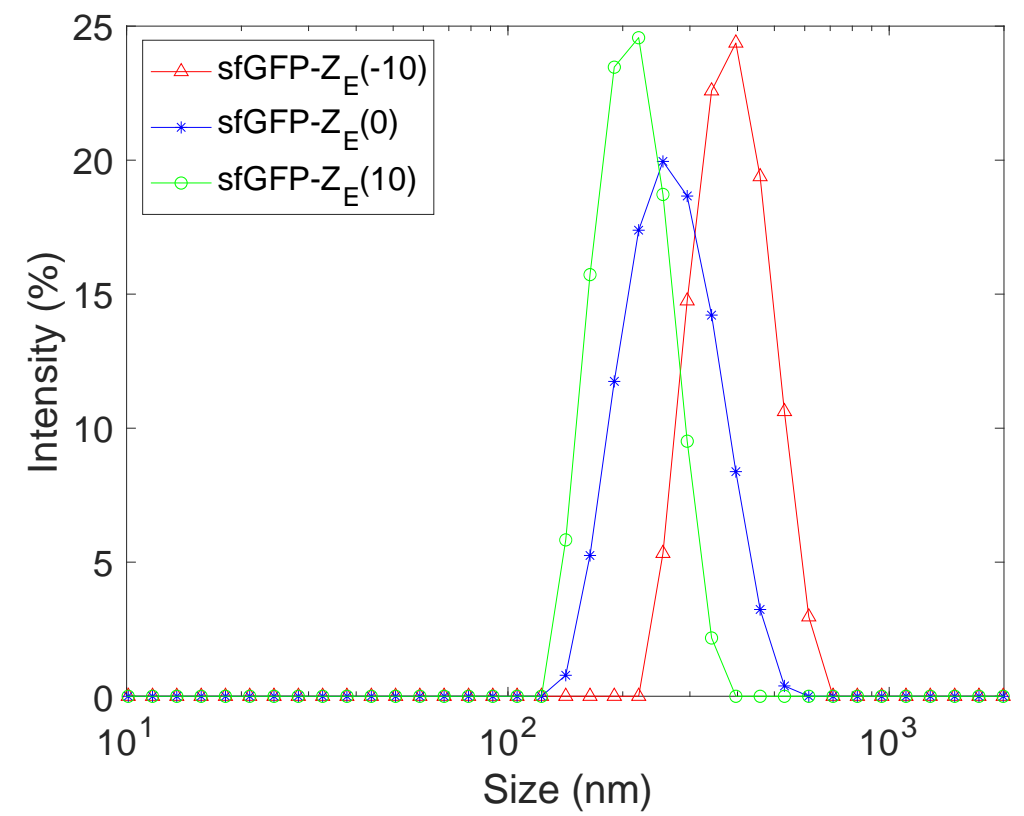

Figure S3. Size intensity plot of sfGFP- $Z_{\mathrm{E}}$ vesicles assembled 1.5 M PBS, $30 \mu \mathrm{M} \mathrm{Z}_{\mathrm{R}}$-ELP, and $Z_{E} / Z_{R}$ of 0.10 . Increasing the salt concentration decreases the size of vesicles from greater than 1 $\mu \mathrm{m}$ to $\sim 400 \mathrm{~nm}$.
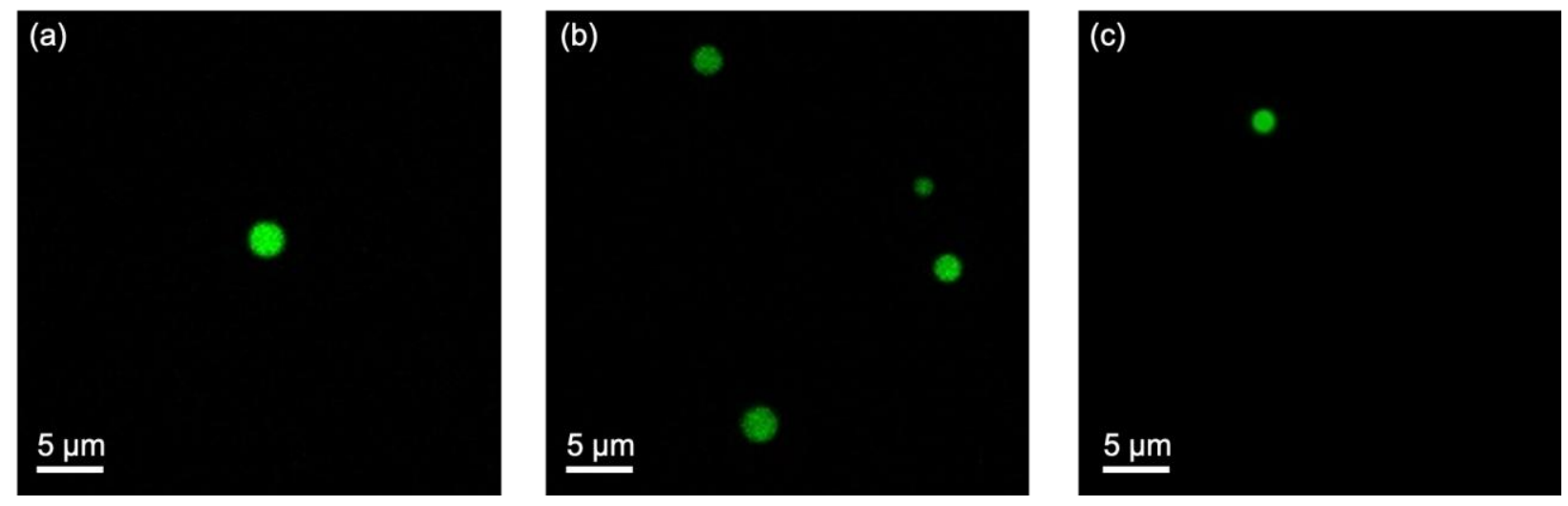

Figure S4. Fluorescent micrographs of $\mathrm{HCA}-\mathrm{Z}_{\mathrm{E}}$ coacervates. $\mathrm{HCA}-\mathrm{Z}_{\mathrm{E}}$ coacervates made at $25^{\circ} \mathrm{C}$ in solutions containing $0.15 \mathrm{M}$ PBS, $10 \mu \mathrm{M} \mathrm{Z} \mathrm{Z}_{\mathrm{R}}-\mathrm{ELP}$, and (a) 0.01 (b) 0.05 (c) $0.10 \mathrm{Z}_{\mathrm{E}} / \mathrm{Z}_{\mathrm{R}}$ molar ratio. 

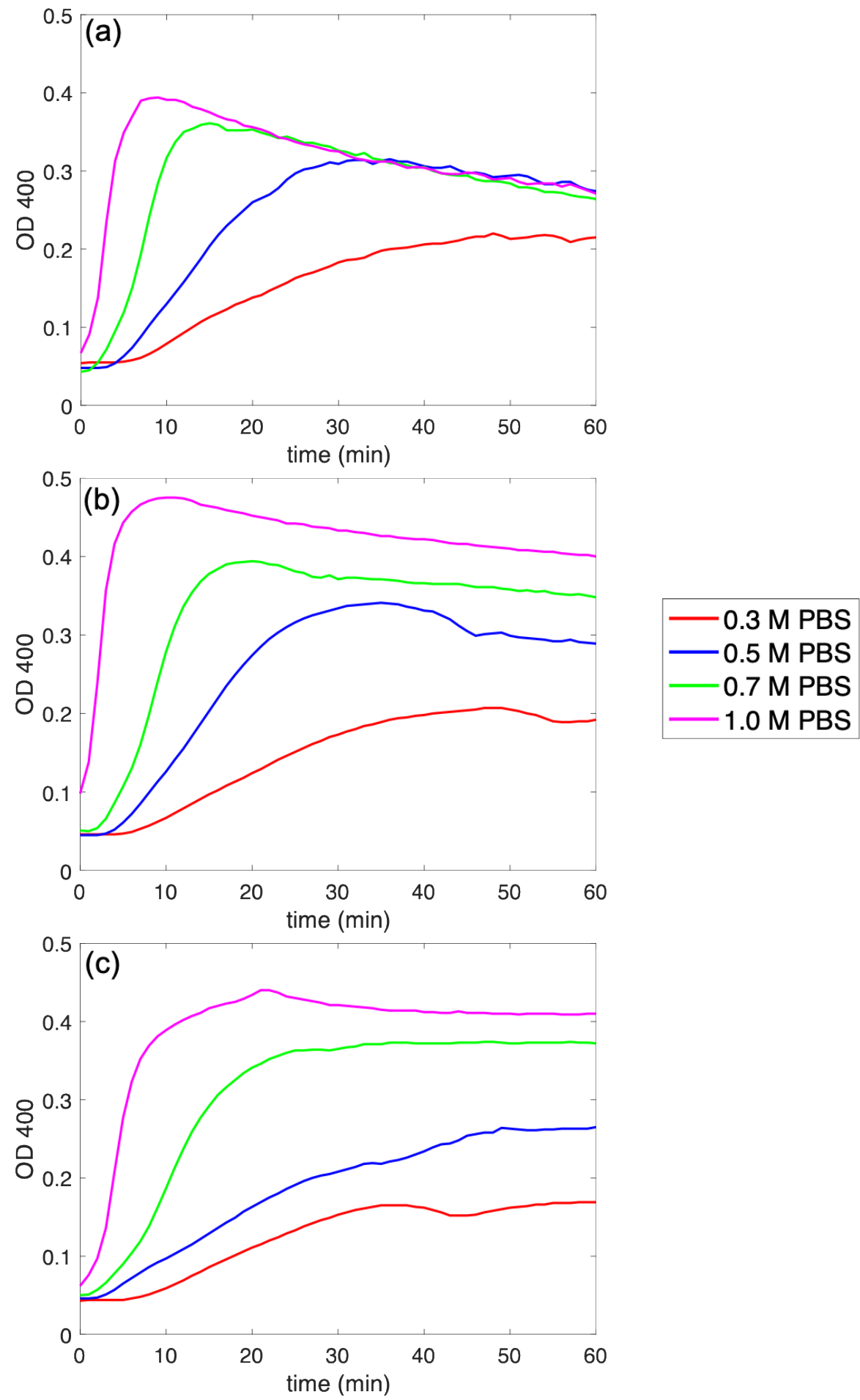

Figure S5. HCA- $Z_{\mathrm{E}}$ turbidity profiles of solutions containing $10 \mu \mathrm{M} \mathrm{Z}_{\mathrm{R}}-\mathrm{ELP}$ with varying $\mathrm{Z}_{\mathrm{E}} / \mathrm{Z}_{\mathrm{R}}$. (a) The turbidity decreases over time for solutions containing $0.01 \mathrm{Z}_{\mathrm{E}} / \mathrm{Z}_{\mathrm{R}}$, signaling the formation of unstable structures. (b) As salt concentration increases, the structures with $0.05 \mathrm{Z}_{\mathrm{E}} / \mathrm{Z}_{\mathrm{R}}$ become more stable. (c) Stable HCA- $Z_{E}$ vesicles with $0.1 Z_{E} / Z_{R}$ form from solutions containing salt concentrations above $0.3 \mathrm{M}$ PBS. 


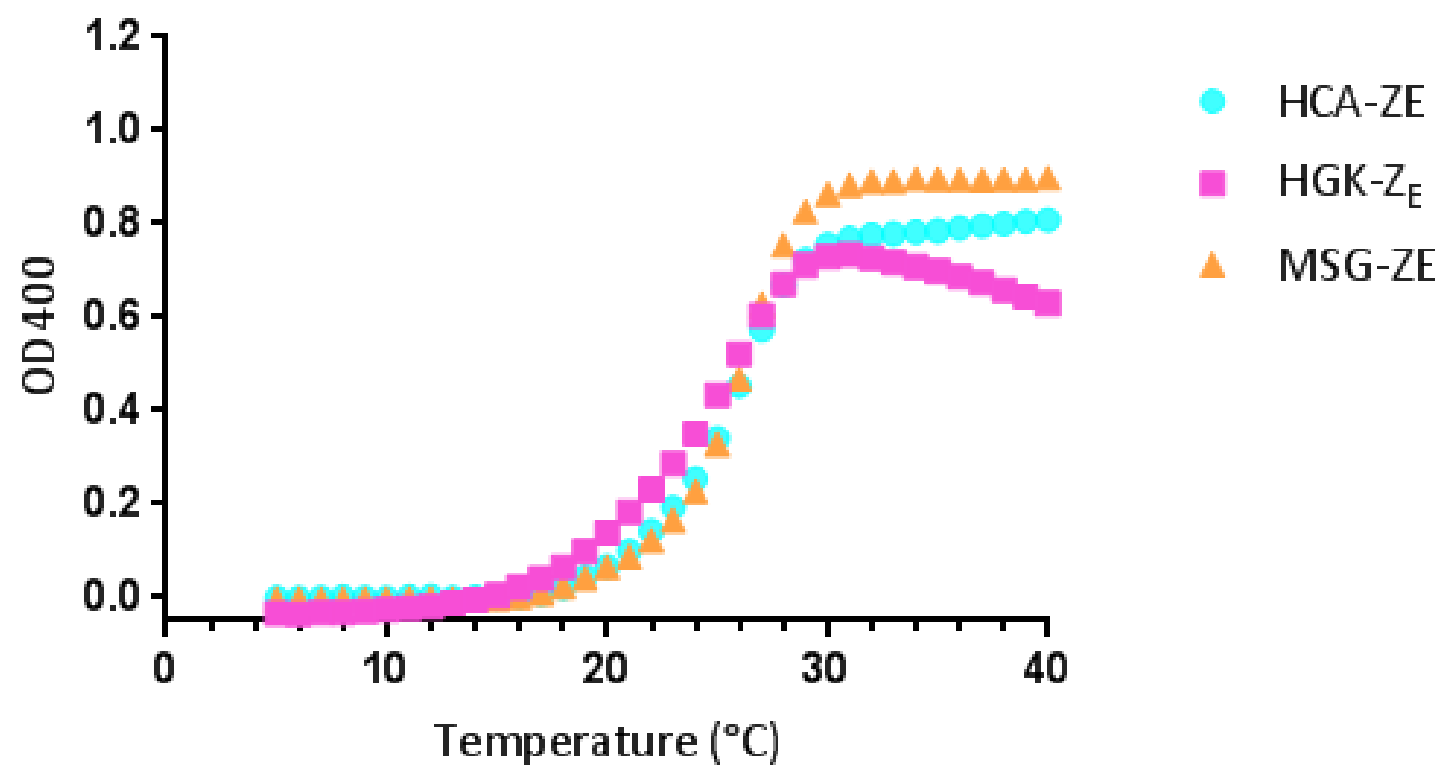

Figure S6. The transition temperature of solutions containing $30 \mu \mathrm{M} \mathrm{Z}_{\mathrm{R}}$-ELP, $1.5 \mu \mathrm{M}$ Globule$\mathrm{Z}_{\mathrm{E}}$, and $0.3 \mathrm{M}$ PBS. The $\mathrm{T}_{\mathrm{t}}$ of HCA-Z $\mathrm{E}, \mathrm{HGK}-\mathrm{Z}_{\mathrm{E}}$, and MSG-Z $\mathrm{Z}_{\mathrm{E}}$ is $27{ }^{\circ} \mathrm{C}, 25^{\circ} \mathrm{C}$ and $27{ }^{\circ} \mathrm{C}$, respectively.

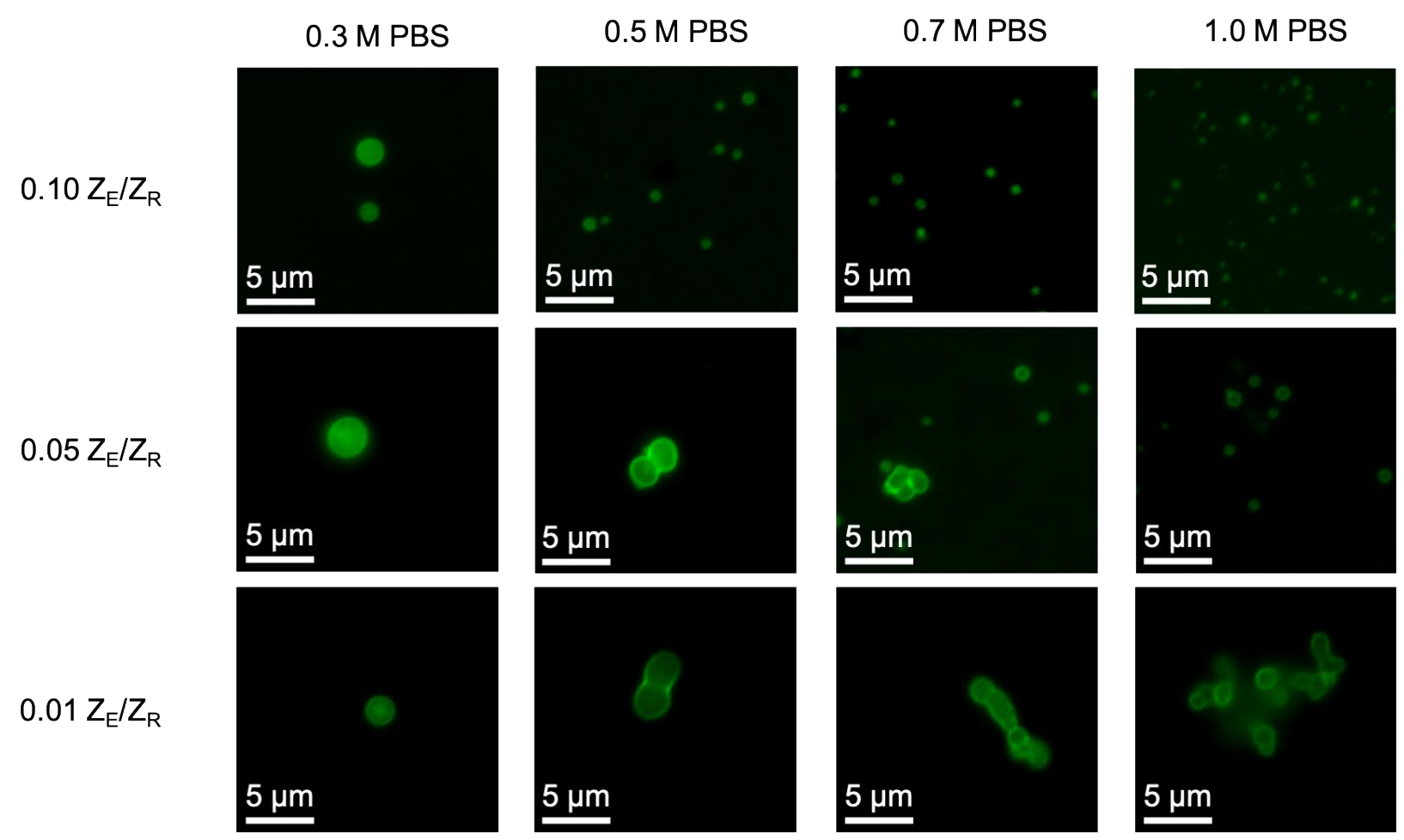

Figure S7. Epifluorescent micrographs of structures in the $\mathrm{HCA}-\mathrm{Z}_{\mathrm{E}}$ phase diagram containing 10 $\mu \mathrm{M} Z_{\mathrm{R}}-\mathrm{ELP}$. 

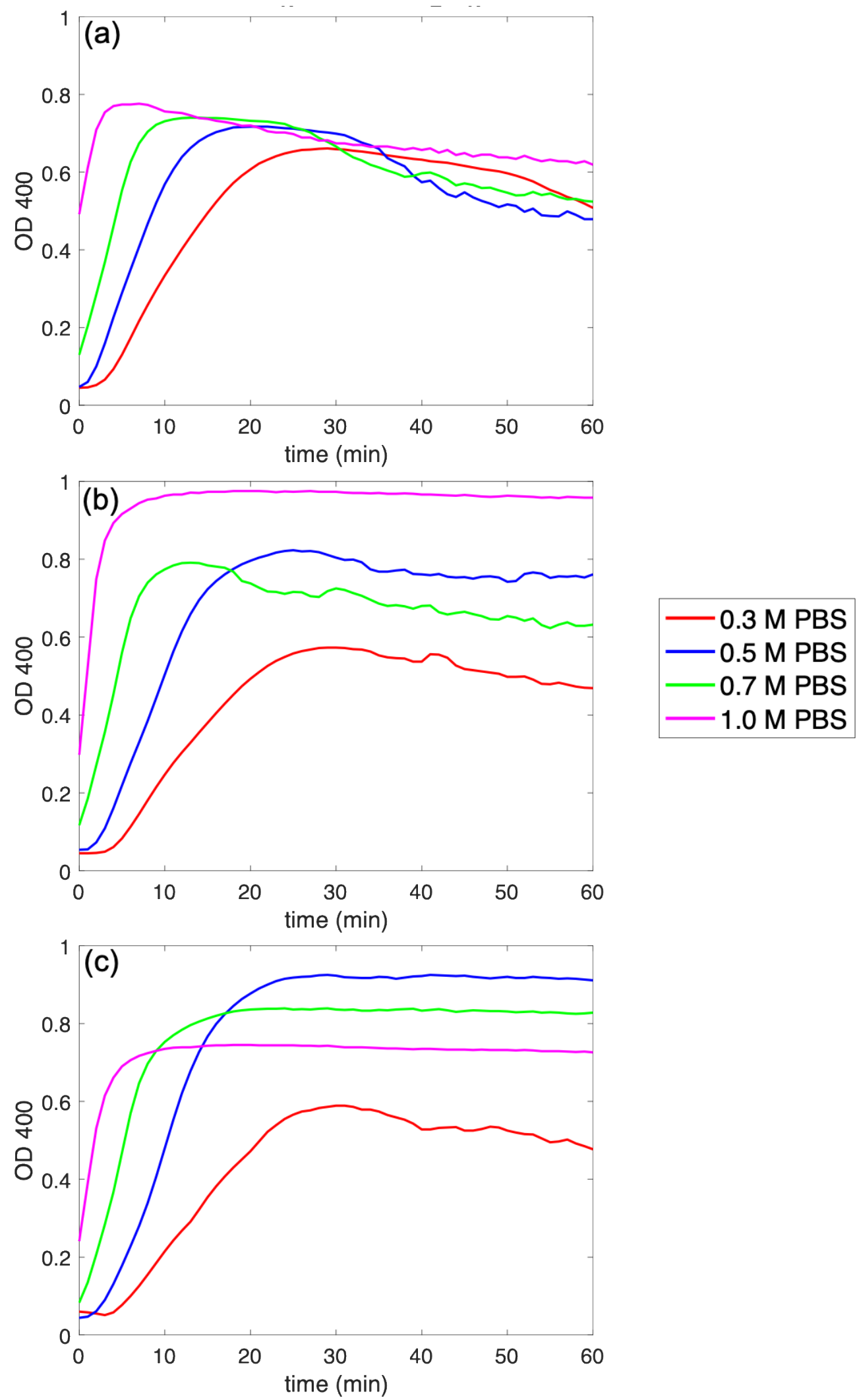

Figure S8. Turbidity profiles of structures assembled at $25{ }^{\circ} \mathrm{C}$ from $\mathrm{HCA}-\mathrm{Z}_{\mathrm{E}}$ with $30 \mu \mathrm{M} \mathrm{Z}_{\mathrm{R}}$-ELP at (a) $0.01,(b), 0.05$, and (c) $0.10 \mathrm{Z}_{\mathrm{E}} / \mathrm{Z}_{\mathrm{R}}$ ratio. 


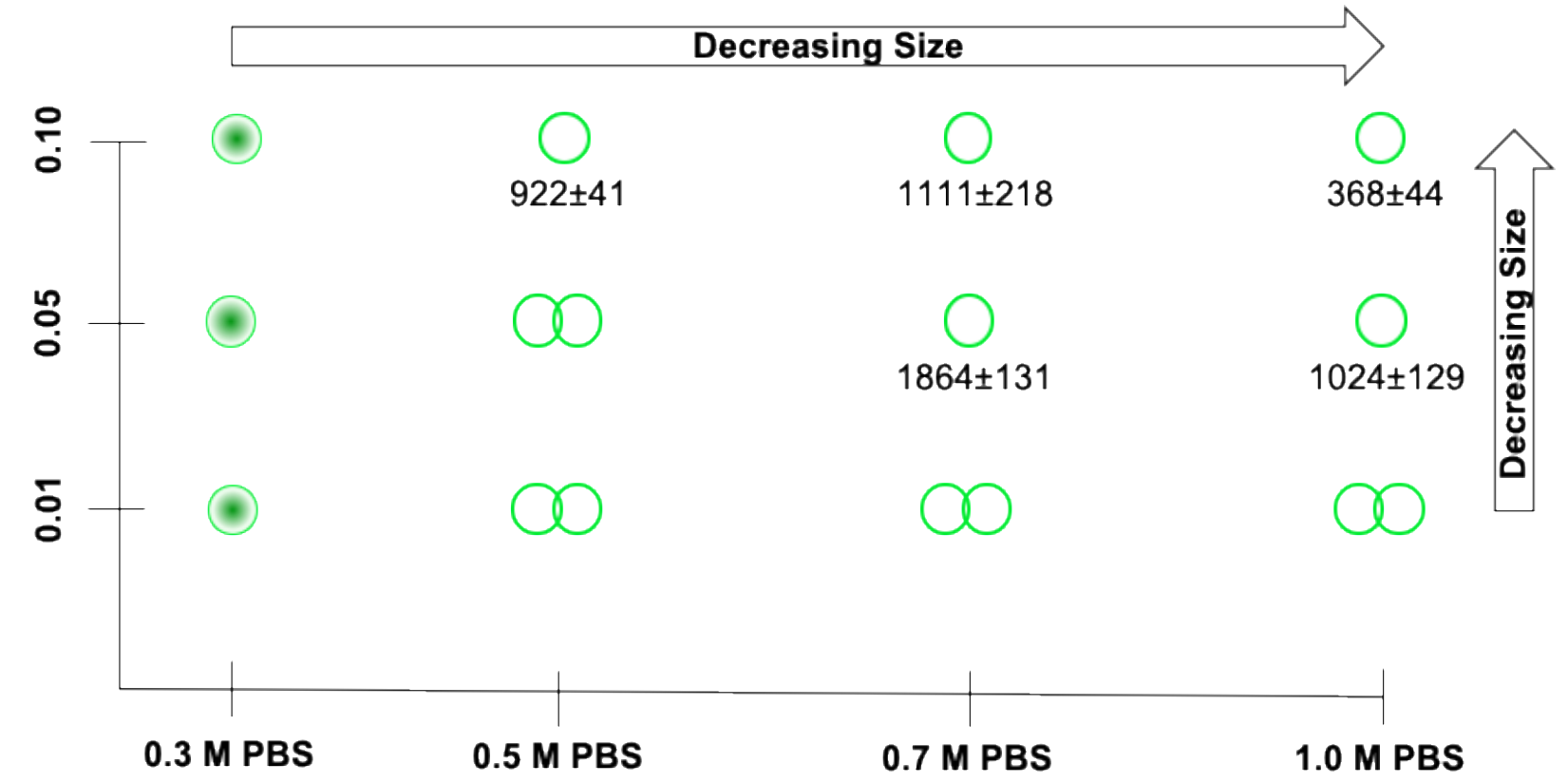

Figure S9. Phase diagram of structures assembled from $\mathrm{HCA}-\mathrm{Z}_{\mathrm{E}}$ at $25{ }^{\circ} \mathrm{C}$ with $30 \mu \mathrm{M} \mathrm{Z}_{\mathrm{R}}-\mathrm{ELP}$. Partially filled circles represent the formation of coacervate-vesicle hybrid structures. Fused rings represent the formation of fused vesicles. Hollow circles represent the formation of vesicles. The average hydrodynamic diameters and standard deviation of stable structures are shown in $\mathrm{nm}$.

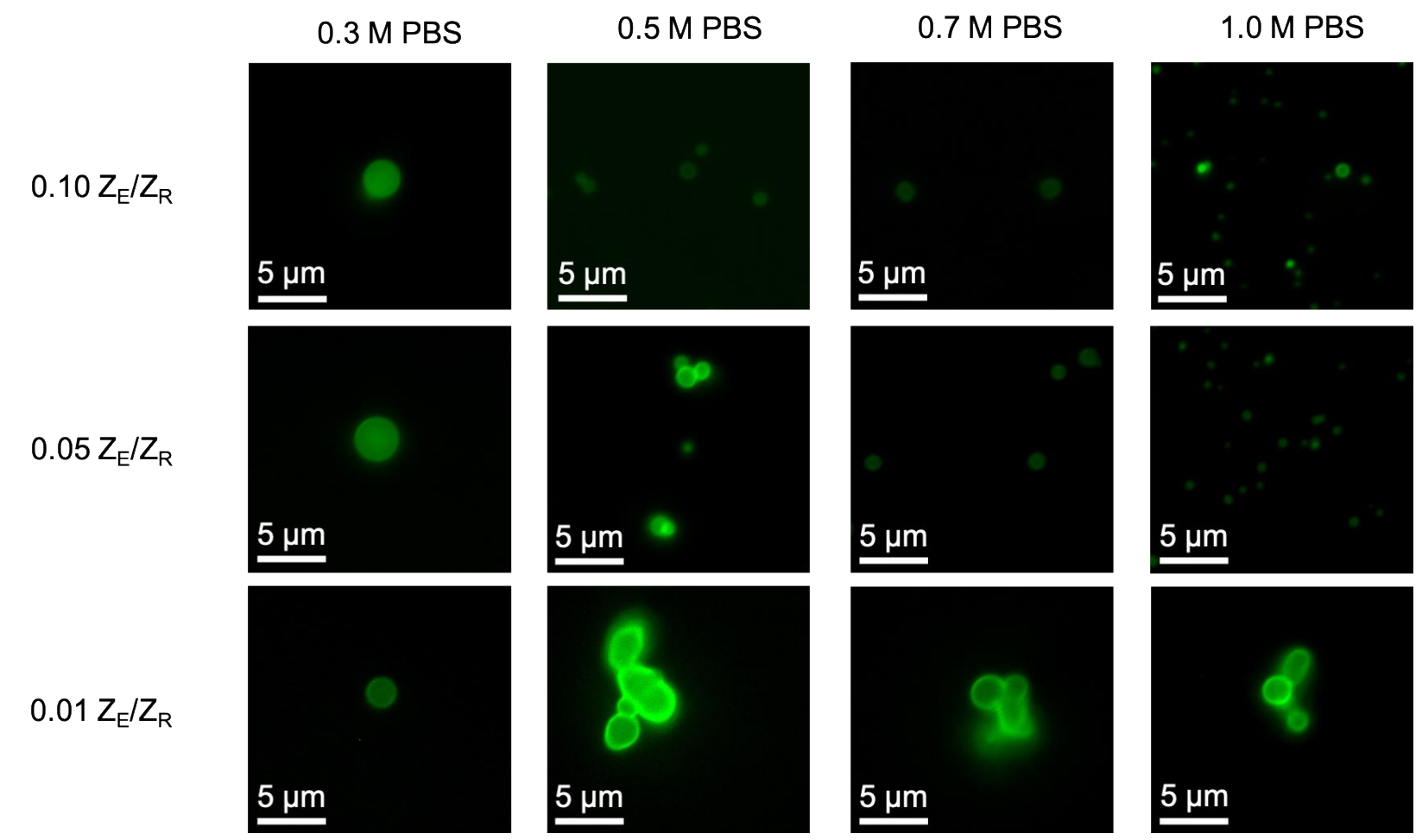

Figure S10. Epifluorescent micrographs of structures in phase diagram assembled from $\mathrm{HCA}-\mathrm{Z}_{\mathrm{E}}$ at $25{ }^{\circ} \mathrm{C}$ with $30 \mu \mathrm{M} \mathrm{Z} \mathrm{Z}_{\mathrm{R}}$-ELP. 


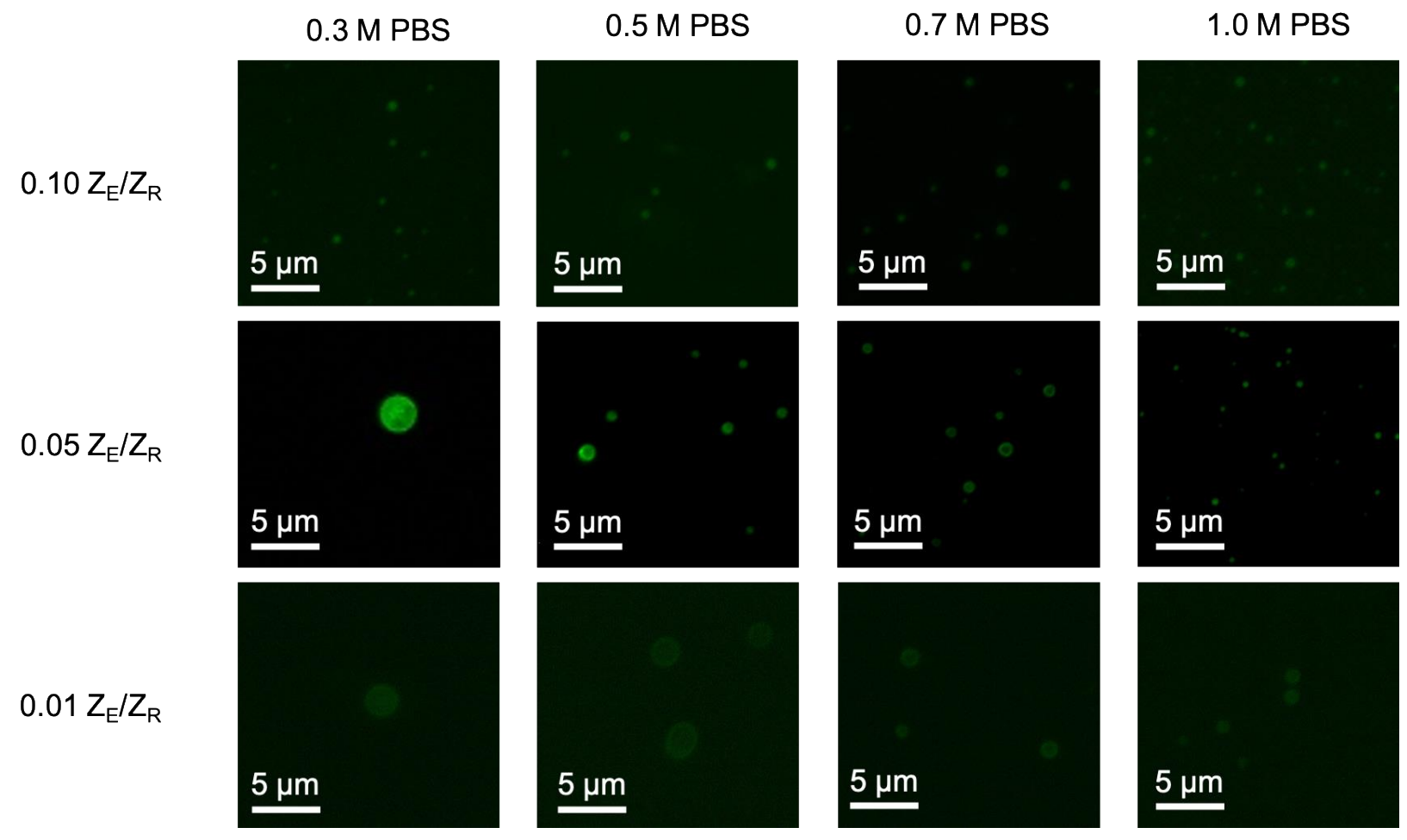

Figure S11. Epifluorescent micrographs of structures in the MSG-ZE phase diagram at $25^{\circ} \mathrm{C}$ with $10 \mu \mathrm{M} \mathrm{Z}_{\mathrm{R}}-\mathrm{ELP}$. 

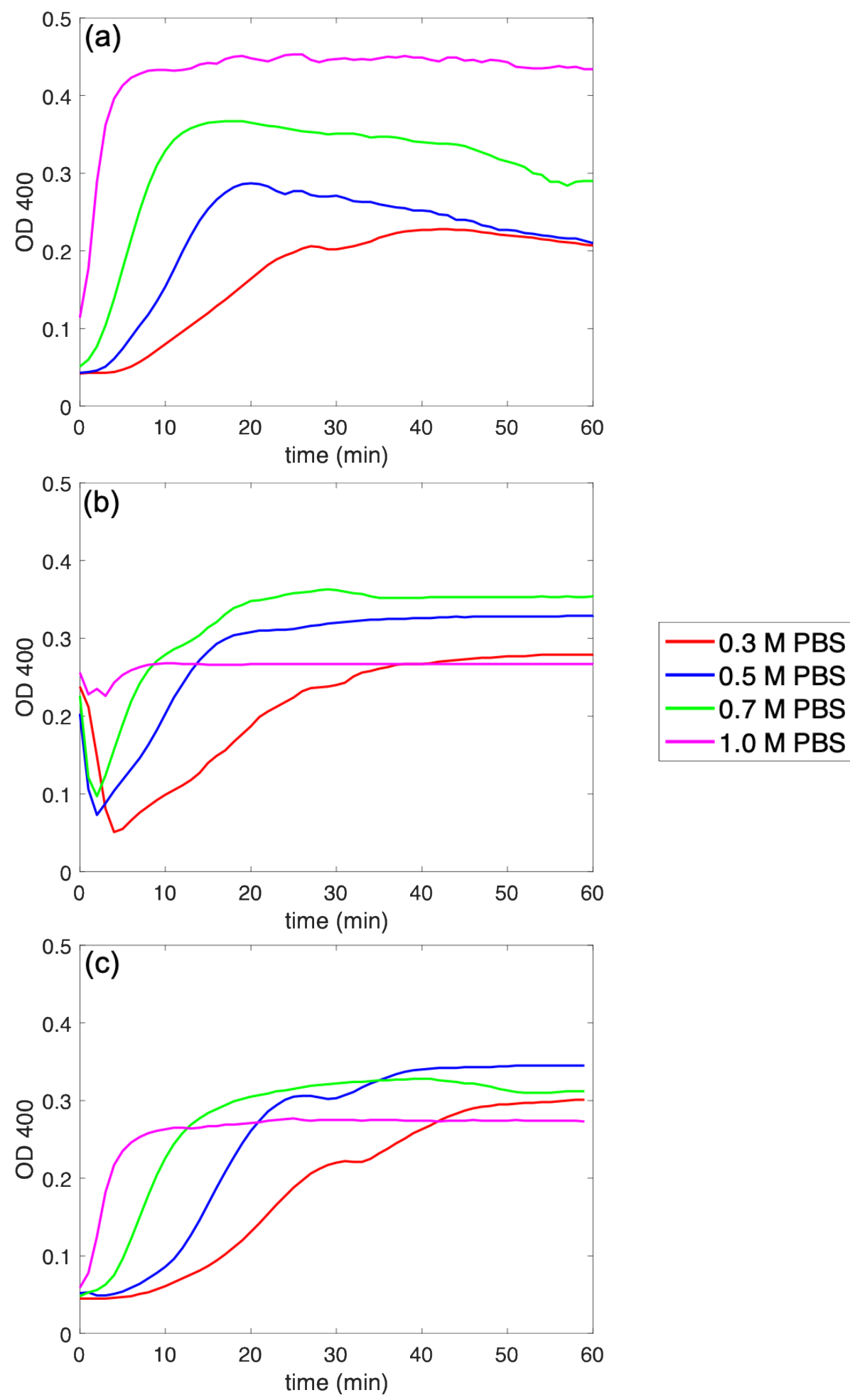

Figure S12. MSG-Z $Z_{E}$ turbidity profiles of solutions containing $10 \mu \mathrm{M} \mathrm{Z}_{\mathrm{R}}-\mathrm{ELP}$. (a) The turbidity decreases over time for solutions containing $0.01 \mathrm{Z}_{\mathrm{E}} / \mathrm{Z}_{\mathrm{R}}$, signaling the formation of unstable structures except at 1.0 M PBS. (b) As $Z_{\mathrm{E}} / \mathrm{Z}_{\mathrm{R}}$ increases to 0.05 and salt concentration increases, the structures become more stable. (c) Stable HCA-Z $Z_{E}$ vesicles form from solutions of all salt concentrations above $0.10 \mathrm{Z}_{\mathrm{E}} / \mathrm{Z}_{\mathrm{R}}$. 

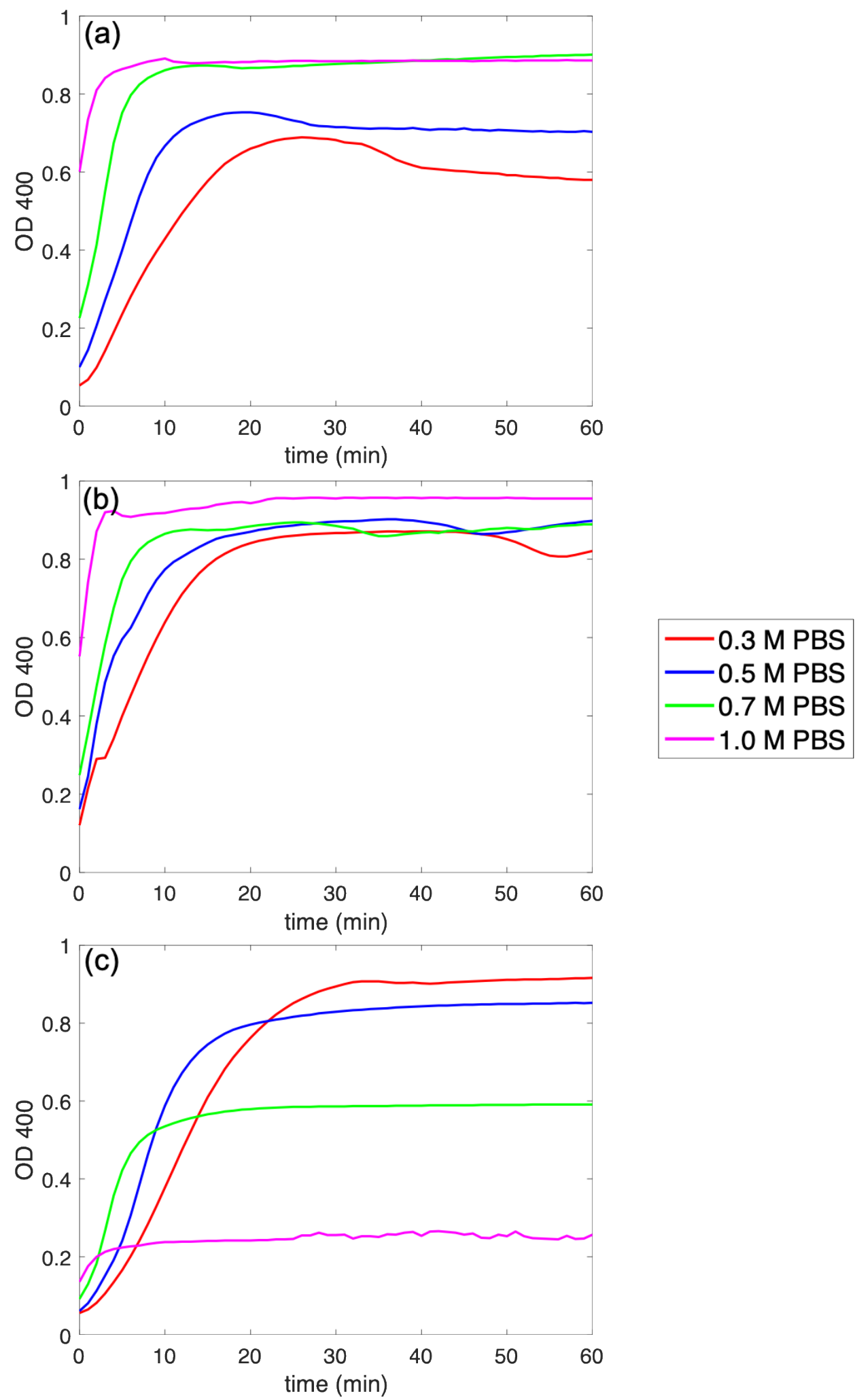

Figure S13. Turbidity profiles of structures assembled from MSG-Z $Z_{E}$ at $25{ }^{\circ} \mathrm{C}$ with $30 \mu \mathrm{M} \mathrm{Z}_{\mathrm{R}^{-}}$ ELP at (a) 0.01, (b), 0.05, and (c) $0.10 \mathrm{Z}_{\mathrm{E}} / \mathrm{Z}_{\mathrm{R}}$ ratio. 


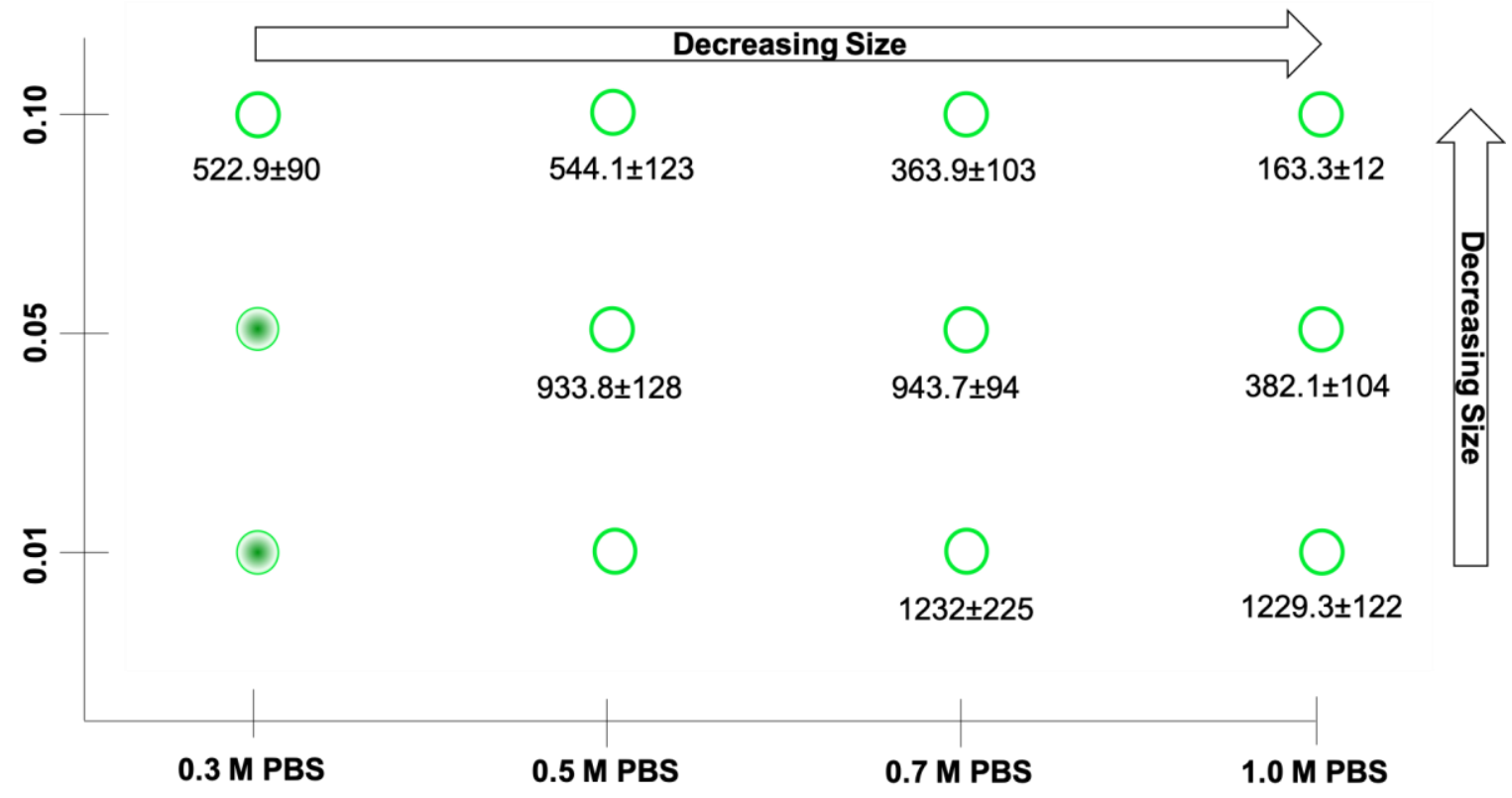

Figure S14. Phase diagram of structures assembled from MSG-Z $Z_{E}$ at $25^{\circ} \mathrm{C}$ with $30 \mu \mathrm{M} \mathrm{Z}_{\mathrm{R}}-\mathrm{ELP}$. Partially filled circles represent coacervate-vesicle hybrids. Hollow circles represent the formation of vesicles. The average hydrodynamic diameters and standard deviation of stable structures are shown in $\mathrm{nm}$.

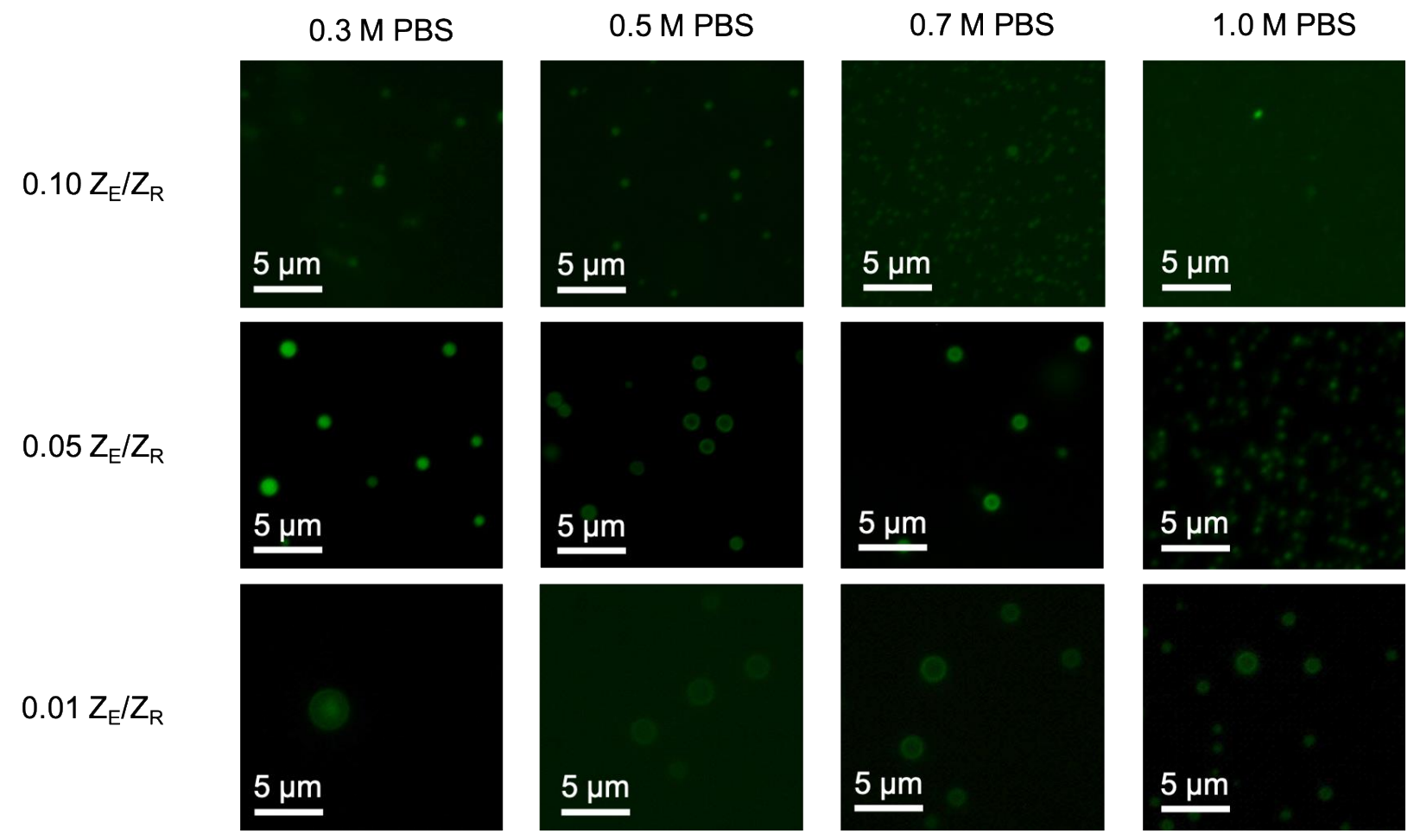

Figure S15. Epifluorescent micrographs of structures in phase diagram assembled from MSG-Z $\mathrm{Z}_{\mathrm{E}}$ at $25^{\circ} \mathrm{C}$ with $30 \mu \mathrm{M} \mathrm{Z} \mathrm{Z}_{\mathrm{R}}$-ELP. 


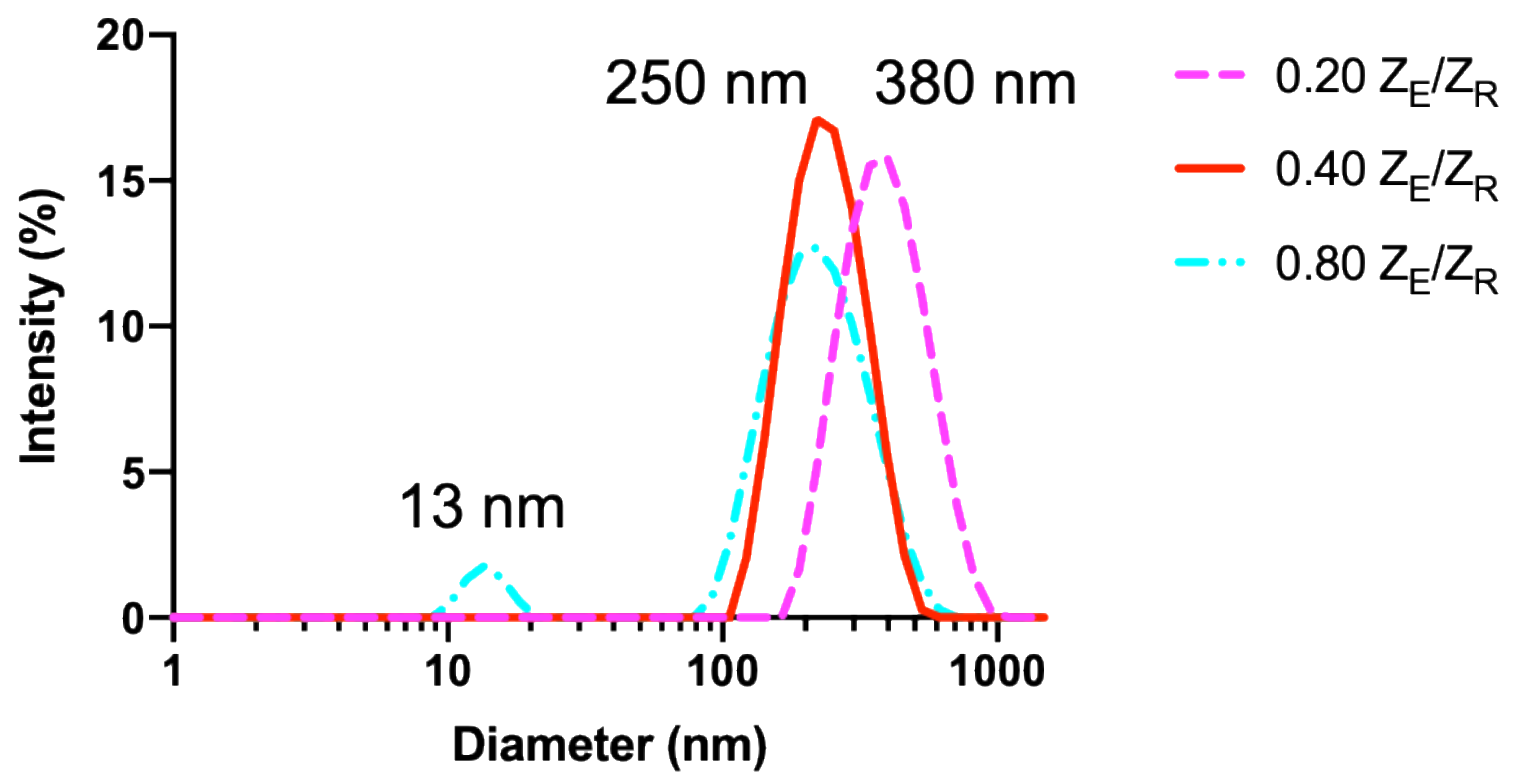

Figure S16. DLS histogram of MSG- $Z_{E}$ vesicles with high $Z_{E} / Z_{R}$ ratio formed with $10 \mu M Z_{R^{-}}$ ELP. Increasing the $Z_{\mathrm{E}} / Z_{\mathrm{R}}$ ratio decreases the size of vesicles until at least $0.40 \mathrm{Z}_{\mathrm{E}} / Z_{\mathrm{R}}$. Above 0.80 $Z_{\mathrm{E}} / Z_{\mathrm{R}}$, smaller structures are not formed, instead there is a soluble peak and a vesicle peak.

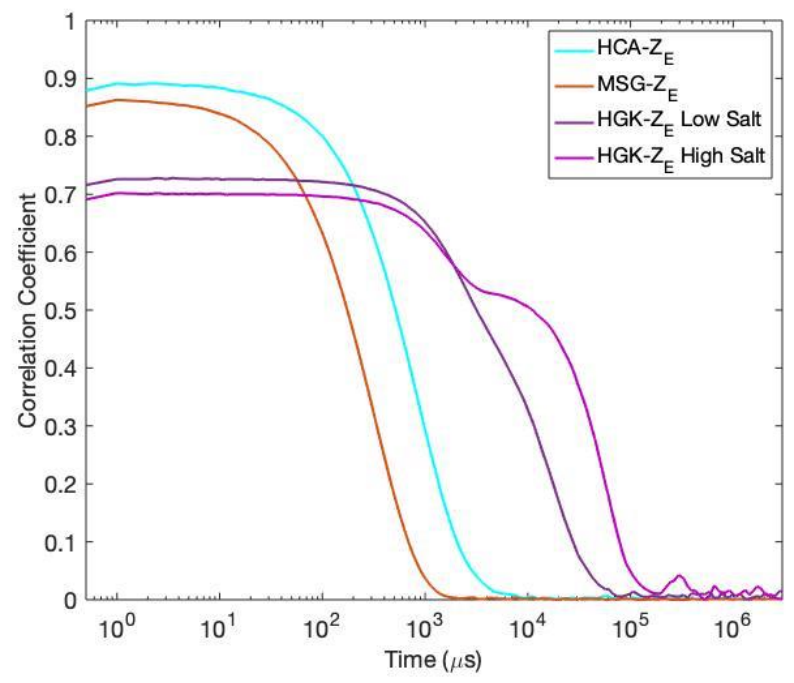

Figure S17- DLS correlograms for solutions containing $10 \mu \mathrm{M} \mathrm{Z}_{\mathrm{R}}-\mathrm{ELP}$ and $0.10 \mathrm{Z}_{\mathrm{E}} / \mathrm{Z}_{\mathrm{R}}$ incubated at $25{ }^{\circ} \mathrm{C}$ for 1 hour. Good quality correlograms are shown for $\mathrm{HCA}-\mathrm{Z}_{\mathrm{E}}$ and $\mathrm{MSG}-\mathrm{Z}_{\mathrm{E}}$ in $1.0 \mathrm{M}$ PBS. Poor quality correlograms are shown for $\mathrm{HGK}-\mathrm{Z}_{\mathrm{E}}$ at 0.5 and 1.0 M PBS. The good quality correlograms have a high correlation coefficient, a steep slope, and a correlation coefficient of zero at high correlation times, which are indicative of low polydispersity and little sedimentation of stable vesicles. The correlograms for poor quality DLS results from $\mathrm{HGK}-\mathrm{Z}_{\mathrm{E}}$ have a gentler slope that spikes up and down at high correlation times, indicative of high polydispersity and sedimentation. 


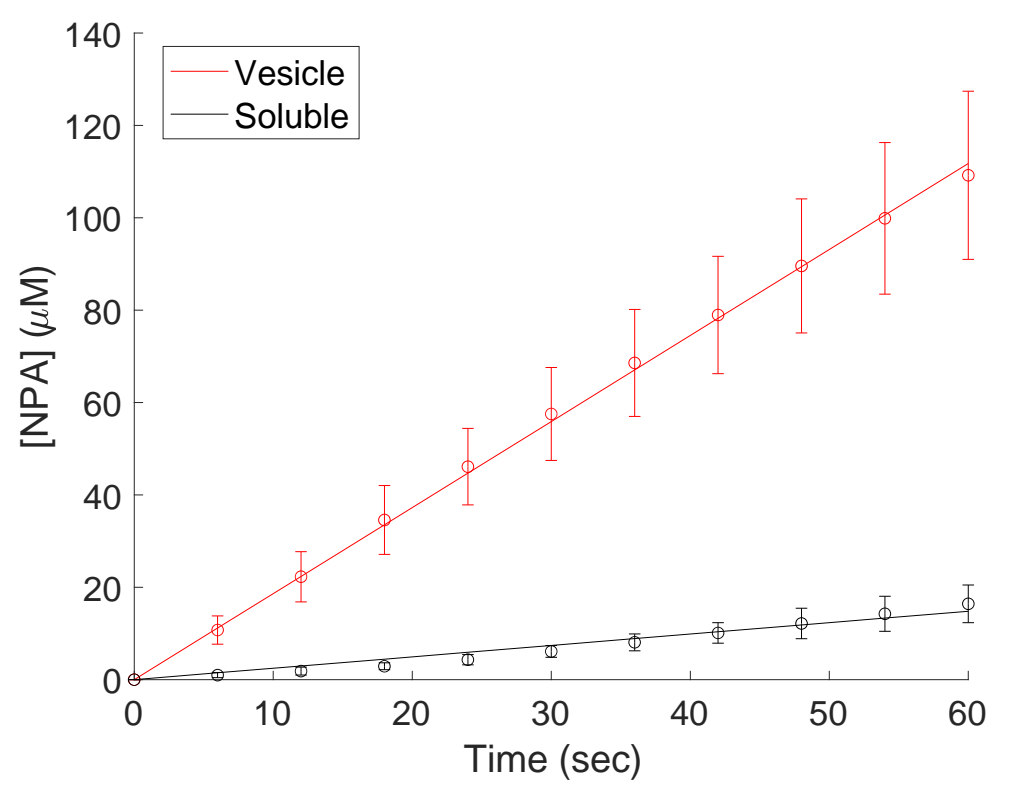

Figure S18. The activity of $\mathrm{HCA}-\mathrm{Z}_{\mathrm{E}}$ was assayed at $30{ }^{\circ} \mathrm{C}$ in the presence of $10 \mathrm{mM} \mathrm{ZnSO}$, and $1 \mathrm{mM}$ 4-nitrophenyl acetate. The specific activity of $\mathrm{HCA}-\mathrm{Z}_{\mathrm{E}}$ under these conditions was $0.34 \pm$ 0.01 and $2.23 \pm 0.38 \mu \mathrm{mol}$ product $/ \mathrm{min} / \mathrm{mg}$ enzyme for the soluble enzyme and vesicles, respectively.

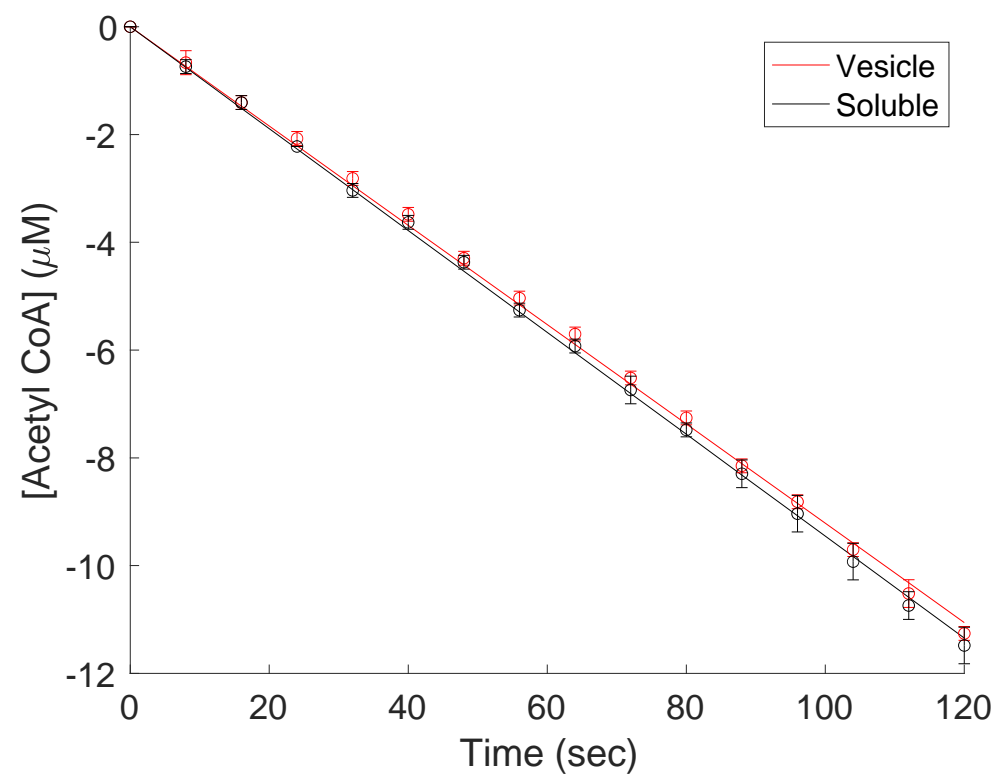

Figure S19. Activity of MSG- $\mathrm{Z}_{\mathrm{E}}$ at $30{ }^{\circ} \mathrm{C}$ in the presence of $5 \mathrm{mM} \mathrm{MgCl}, 0.25 \mathrm{mM}$ acetyl CoA, and $1 \mathrm{mM}$ glyoxylate. The specific activity of $M S G-Z_{E}$ under these conditions was $4.53 \pm 0.13$ and $4.42 \pm 0.06 \mu \mathrm{mol} \mathrm{product} / \mathrm{min} / \mathrm{mg}$ enzyme for the soluble enzyme and vesicles, respectively. 


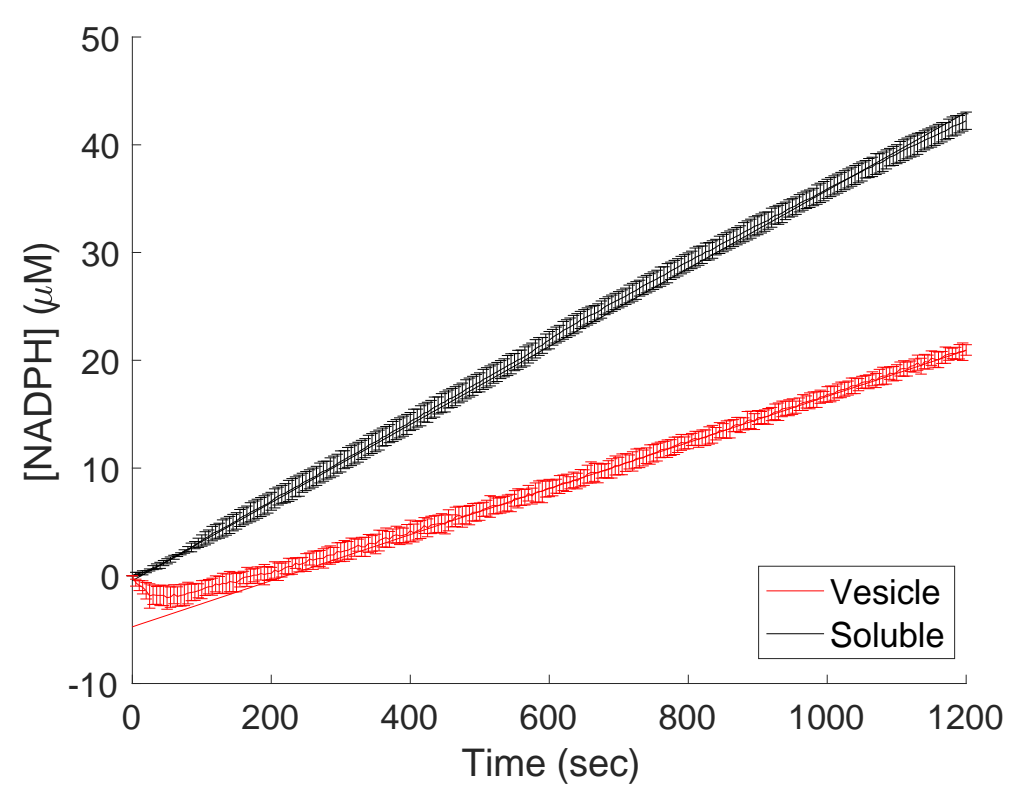

Figure S20. Activity of $\mathrm{HGK}-\mathrm{Z}_{\mathrm{E}}$ at $30{ }^{\circ} \mathrm{C}$ in the presence of $50 \mathrm{mM}$ glucose, $1 \mathrm{mM}$ ATP, and 5 $\mathrm{mM} \mathrm{MgCl}$. To detect HGK-Z $\mathrm{E}_{\mathrm{E}}$ activity, two units of glucose-6-phosphate dehydrogenase and 0.5 mM NADPH were added to the solution to obtain an absorbance reading for NADPH. The specific activity of HGK-Z $\mathrm{E}_{\mathrm{E}}$ under these conditions was $0.10 \pm 0.002$ and $0.06 \pm 0.0006 \mu \mathrm{mol}$ product $/ \mathrm{min} / \mathrm{mg}$ enzyme for the soluble enzyme and vesicles, respectively.
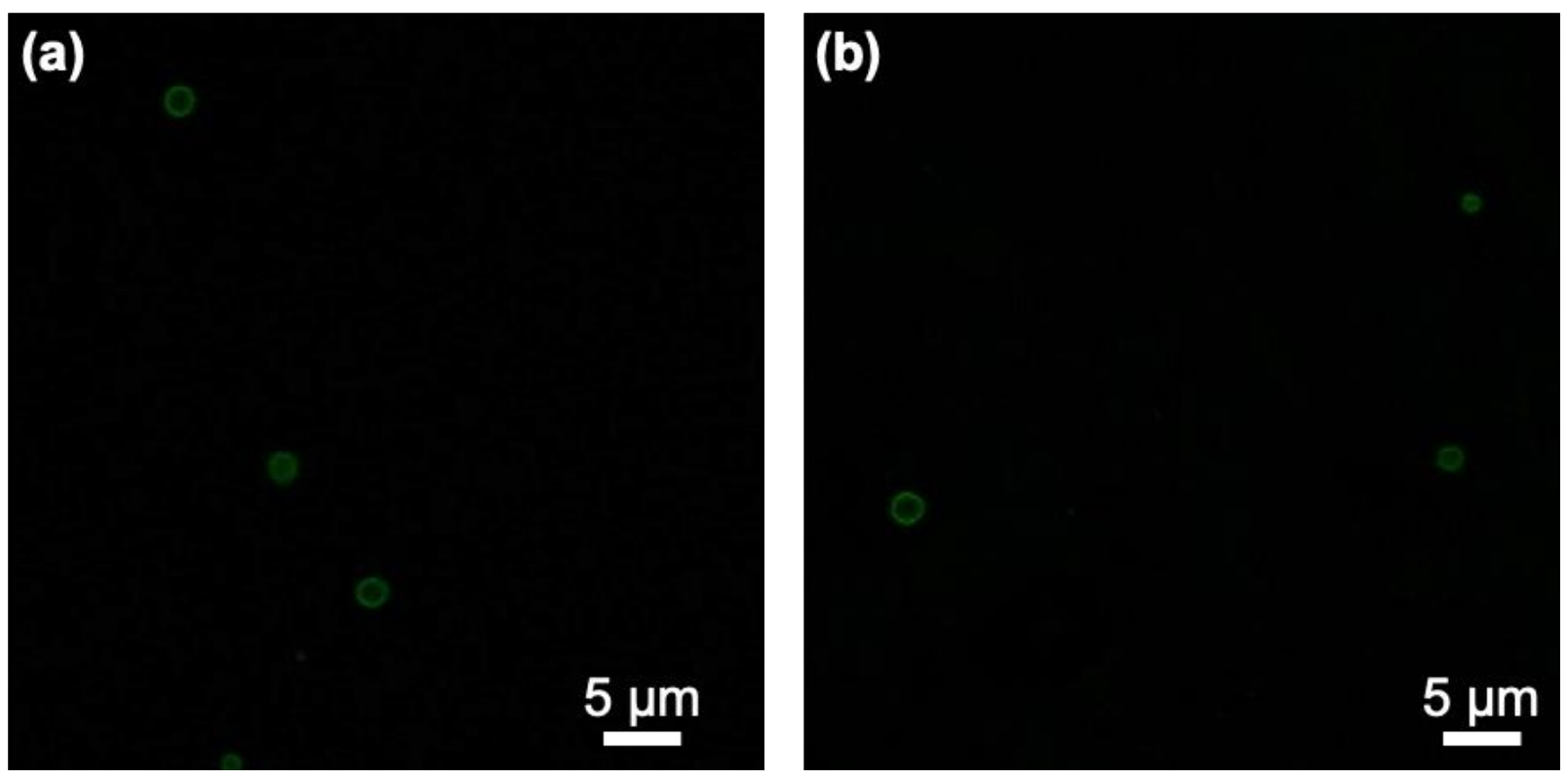

Figure S21. Effect of presence of hydrophobic substrate on $\mathrm{HCA}-\mathrm{Z}_{\mathrm{E}}$ vesicles. (a) $\mathrm{HCA}-\mathrm{Z}_{\mathrm{E}}$ vesicles incubated with $1 \mathrm{mM}$ 4-nitrophenyl acetate and $10 \mathrm{mM} \mathrm{ZnSO} 4$ after 1 hour of incubation under reaction conditions. (b) $\mathrm{HCA}-\mathrm{Z}_{\mathrm{E}}$ vesicles incubated under reaction conditions without 4nitrophenyl acetate substrate after 1 hour. 


\begin{tabular}{|c|c|c|c|}
\hline sfGFP-ZE Variant & -10 & 0 & +10 \\
\hline Zeta Potential (mV) & $-15.7 \pm 1.1$ & $-10.1 \pm 0.8$ & $9.32 \pm 0.1$ \\
\hline
\end{tabular}

Table S1. Zeta potential values for soluble sfGFP-Z $Z_{E}(-10), \operatorname{sfGFP}-Z_{E}(0)$, and $s f G F P-Z_{E}(10)$. The values for each variant are lower than the sfGFP alone because they are bound to a negatively charged glutamate rich leucine zipper.

DNA and amino acid sequences for all 7 proteins used in this work. The altered amino acids for sfGFP are highlighted in red for positive changes and blue for negative changes.

\begin{tabular}{|c|c|c|}
\hline Protein & DNA Sequence & $\begin{array}{c}\text { Amino Acid } \\
\text { Sequence }\end{array}$ \\
\hline$Z_{R}-E L P$ & $\begin{array}{l}\text { ATGAAAGGATCCCTGGAAATCCGTGCGGCGGCGCTGCGTC } \\
\text { GTCGTAACACCGCGCTGCGTACCCGTGTTGCGGAACTGCGT } \\
\text { CAGCGTGTTCAGCGTCTGCGTAACGAAGTTTCTCAGTACGA } \\
\text { AACCCGTTACGGTCCGCTGGGTGGTGGCGGTTCTGGTGGC } \\
\text { GGTGGATCCGGGGTGCCGGGTGTGGGCGTTCCGGGCGTG } \\
\text { GGTGTACCGGGCTTCGGTGTCCCGGGCGTAGGTGTTCCGG } \\
\text { GTGTCGGGGTGCCGGGTGTGGGCGTTCCGGGCGTGGGTG } \\
\text { TACCGGGCTTCGGTGTCCCGGGCGTAGGTGTTCCGGGTGT } \\
\text { CGGGGTGCCGGGTGTGGGCGTTCCGGGCGTGGGTGTACC } \\
\text { GGGCTTCGGTGTCCCGGGCGTAGGTGTTCCGGGTGTCGGG } \\
\text { GTGCCGGGTGTGGGCGTTCCGGGCGTGGGTGTACCGGGCT } \\
\text { TCGGTGTCCCGGGCGTAGGTGTTCCGGGTGTCGGGGTGCC } \\
\text { GGGTGTGGGCGTTCCGGGCGTGGGTGTACCGGGCTTCGGT } \\
\text { GTCCCGGGCGTAGGTGTTCCGGGTGTCGGGGTGCCCGGGT } \\
\text { GCTAAA }\end{array}$ & $\begin{array}{l}\text { MKGSLEIRAAALRR } \\
\text { RNTALRTRVAELRQ } \\
\text { RVQRLRNEVSQYET } \\
\text { RYGPLGGGGSGGG } \\
\text { GSGVPGVGVPGVG } \\
\text { VPGFGVPGVGVPG } \\
\text { VGVPGVGVPGVGV } \\
\text { PGFGVPGVGVPGV } \\
\text { GVPGVGVPGVGVP } \\
\text { GFGVPGVGVPGVG } \\
\text { VPGVGVPGVGVPG } \\
\text { FGVPGVGVPGVGV } \\
\text { PGVGVPGVGVPGF } \\
\text { GVPGVGVPGVGVP } \\
\text { GC }\end{array}$ \\
\hline sfGFP-ZE $(-10)$ & $\begin{array}{l}\text { ATGGGCCACCACCACCACCACCACCGTAGCAGCAAAGGCG } \\
\text { AGGAACTGTTCACCGGTGTGGTTCCGATCCTGGTGGAACTG } \\
\text { GACGGCGATGTTAACGGTCACAAGTTTAGCGTTCGTGGTG } \\
\text { AGGGCGAAGGTGACGCGACCAACGGCAAGCTGACCCTGA } \\
\text { AATTCATTTGCACCACCGGTAAACTGCCGGTGCCGTGGCCG } \\
\text { ACCCTGGTTACCACCCTGACCTACGGCGTTCAGTGCTTTAGC } \\
\text { CGTTATCCGGACCACATGAAGCGTCACGATTTCTTTAAAG } \\
\text { CGCGATGCCGGAAGGCTACGTGCAAGAGCGTACCATCAGC } \\
\text { TTCAAGGACGATGGTACCTATAAAACCCGTGCGGAAGTTAA } \\
\text { ATTTGAGGGCGACACCCTGGTGAACCGTATCGAACTGAAG } \\
\text { GGTATTGACTTCAAAGAGGATGGCAACATCCTGGGTCACA } \\
\text { AGCTGGAATACAACTTTAACAGCCACAACGTGTATATTACC } \\
\text { GCGGATGAACAGAAAAACGGCATCAAGGCGAACTTTAAAA } \\
\text { TTCGTCACAACGTGGAGGACGGTAGCGTTCAACTGGCGGA } \\
\text { TCACTACCAGCAAAACACCCCGATTGGTGATGGTCCGGTTC } \\
\text { TGCTGCCGGATAACCACTATCTGAGCACCCAGAGCGTGCTG }\end{array}$ & $\begin{array}{l}\text { MGHHHHHHRSSK } \\
\text { GEELFTGVVPILVEL } \\
\text { DGDVNGHKFSVRG } \\
\text { EGEGDATNGKLTLK } \\
\text { FICTTGKLPVPWPT } \\
\text { LVTTLTYGVQCFSR } \\
\text { YPDHMKRHDFFKS } \\
\text { AMPEGYVQERTISF } \\
\text { KDDGTYKTRAEVKF } \\
\text { EGDTLVNRIELKGID } \\
\text { FKEDGNILGHKLEY } \\
\text { NFNSHNVYITADEQ } \\
\text { KNGIKANFKIRHNV } \\
\text { EDGSVQLADHYQQ } \\
\text { NTPIGDGPVLLPDN } \\
\text { HYLSTQSVLSKDPD }\end{array}$ \\
\hline
\end{tabular}




\begin{tabular}{|c|c|c|}
\hline & $\begin{array}{l}\text { AGCAAGGACCCGGATGAAGAACGTGATCACATGGTGCTGC } \\
\text { TGGAGTTCGTTACCGCGGCGGGCATCACCCACGGTATGGA } \\
\text { TGAACTGTACAAACTGCGTGGCAGCGGTAGCCTGGAGATT } \\
\text { GAAGCGGCGGCGCTGGAGCAGGAAAACACCGCGCTGGAG } \\
\text { ACCGAAGTGGCGGAGCTGGAACAGGAAGTGCAACGTCTG } \\
\text { GAGAACATTGTGAGCCAATACCGTACCCGTTATGGTCCGCT } \\
\text { G }\end{array}$ & $\begin{array}{l}\text { EERDHMVLLEFVTA } \\
\text { AGITHGMDELYKLR } \\
\text { GSGSLEIEAAALEQE } \\
\text { NTALETEVAELEQE } \\
\text { VQRLENIVSQYRTR } \\
\text { YGPL }\end{array}$ \\
\hline$s f G F P-Z_{E}(0)$ & $\begin{array}{l}\text { ATGGGCGGCCACCACCACCACCACCACCGTAGCAGCAAAG } \\
\text { GCGAGGAACTGTTCACCGGTGTGGTTCCGATCCTGGTGGA } \\
\text { ACTGGACGGCGATGTTAACGGTCACAAGTTTAGCGTTCGTG } \\
\text { GTGAGGGCGAAGGTGACGCGACCAACGGCAAGCTGACCCT } \\
\text { GAAATTCATTTGCACCACCGGTAAACTGCCGGTGCCGTGGC } \\
\text { CGACCCTGGTTACCACCCTGACCTACGGCGTTCAGTGCTTTA } \\
\text { GCCGTTATCCGGACCACATGAAGCGTCACGATTTCTTTAAA } \\
\text { AGCGCGATGCCGGAAGGCTACGTGCAAGAGCGTACCATCA } \\
\text { GCTTCAAGGACGATGGTACCTATAAAACCCGTGCGGAAGTT } \\
\text { AAATTTGAGGGCGACACCCTGGTGAACCGTATCGAACTGA } \\
\text { AGGGTATTGACTTCAAAAAAAAGGCAACATCCTGGGTCAC } \\
\text { AAGCTGGAATACAACTTTAACAGCCACAACGTGTATATTAC } \\
\text { CGCGGATAAGAAAAAAAACGGCATCAAGGCGAACTTTAAA } \\
\text { ATTCGTCACAACGTGGAGGACGGTAGCGTTCAACTGGCGG } \\
\text { ATCACTACCAGCAAAACACCCCGATTGGTGATGGTCCGGTT } \\
\text { CTGCTGCCGGATAACCACTATCTGAGCACCCAGAGCGTGCT } \\
\text { GAGCAAGGACCCGAAAGAAAAACGTGATCACATGGTGCTG } \\
\text { CTGGAGTTCGTTACCGCGGCGGGCATCACCCACGGTATGG } \\
\text { ATGAACTGTACAAACTGCGTGGCAGCGGTAGCCTGGAGAT } \\
\text { TGAAGCGGCGGCGCTGGAGCAGGAAAACACCGCGCTGGA } \\
\text { GACCGAAGTGGCGGAGCTGGAACAGGAAGTGCAACGTCT } \\
\text { GGAGAACATTGTGAGCCAATACCGTACCCGTTATGGTCCGC } \\
\text { TGTAAAAGCTT }\end{array}$ & $\begin{array}{l}\text { MGHHHHHHRSSK } \\
\text { GEELFTGVVPILVEL } \\
\text { DGDVNGHKFSVRG } \\
\text { EGEGDATNGKLTLK } \\
\text { FICTTGKLPVPWPT } \\
\text { LVTTLTYGVQCFSR } \\
\text { YPDHMKRHDFFKS } \\
\text { AMPEGYVQERTISF } \\
\text { KDDGTYKTRAEVKF } \\
\text { EGDTLVNRIELKGID } \\
\text { FKKKGNILGHKLEY } \\
\text { NFNSHNVYITADKK } \\
\text { KNGIKANFKIRHNV } \\
\text { EDGSVQLADHYQQ } \\
\text { NTPIGDGPVLLPDN } \\
\text { HYLSTQSVLSKDPK } \\
\text { EKRDHMVLLEFTA } \\
\text { AGITHGMDELYKLR } \\
\text { GSGSLEIEAAALEQE } \\
\text { NTALETEVAELEQE } \\
\text { VQRLENIVSQYRTR } \\
\text { YGPL }\end{array}$ \\
\hline sfGFP-ZE $(10)$ & $\begin{array}{l}\text { ATGGGCCACCACCACCACCACCACCGTAGCAGCAAGGGCG } \\
\text { AAAAACTGTTCACCGGTGTGGTTCCGATCCTGGTGGAGCTG } \\
\text { GACGGCGATGTTAACGGTCACAAGTTTAGCGTTCGTGGTG } \\
\text { AGGGCGAAGGTGACGCGACCAACGGCAAGCTGACCCTGA } \\
\text { AATTCATTTGCACCACCGGTAAACTGCCGGTGCCGTGGCCG } \\
\text { ACCCTGGTTACCACCCTGACCTACGGCGTTCAGTGCTTTAGC } \\
\text { CGTTATCCGAAGCACATGAAACGTCACGACTTCTTTAAGAG } \\
\text { CGCGATGCCGGAAGGCTACGTGCAAGAGCGTACCATCAGC } \\
\text { TTCAAGAAAGATGGTACCTATAAAACCCGTGCGGAAGTTAA } \\
\text { ATTTGAGGGCAAAACCCTGGTGAACCGTATCGAACTGAAG } \\
\text { GGTATTGACTTCAAGAAAAAGGGCAACATCCTGGGTCACA } \\
\text { AACTGGAGTACAACTTTAACAGCCACAACGTGTATATTACC } \\
\text { GCGGATAAAAAGAAAAACGGCATCAAGGCGAACTTTAAAA } \\
\text { TTCGTCACAACGTGGAAGACGGTAGCGTTCAGCTGGCGGA }\end{array}$ & $\begin{array}{l}\text { MGHHHHHHRSSK } \\
\text { GEKLFTGVVPILVEL } \\
\text { DGDVNGHKFSVRG } \\
\text { EGEGDATNGKLTLK } \\
\text { FICTTGKLPVPWPT } \\
\text { LVTTLTYGVQCFSR } \\
\text { YPKHMKRHDFFKS } \\
\text { AMPEGYVQERTISF } \\
\text { KKDGTYKTRAEVKF } \\
\text { EGKTLVNRIELKGID } \\
\text { FKKKGNILGHKLEY } \\
\text { NFNSHNVYITADKK } \\
\text { KNGIKANFKIRHNV } \\
\text { EDGSVQLADHYQQ }\end{array}$ \\
\hline
\end{tabular}




\begin{tabular}{|c|c|c|}
\hline & $\begin{array}{l}\text { TCACTACCAGCAAAACACCCCGATCGGCAAGGGTCCGGTTC } \\
\text { TGCTGCCGGACAACCACTATCTGAGCACCCAAAGCGTGCTG } \\
\text { AGCAAAGACCCGAAGGAAAAACGTGATCACATGGTGCTGC } \\
\text { TGGAGTTCGTTACCGCGGCGGGCATCACCCACGGTATGGA } \\
\text { TGAACTGTACAAGCTGCGTGGCAGCGGTAGCCTGGAGATT } \\
\text { GAAGCGGCGGCGCTGGAGCAGGAAAACACCGCGCTGGAG } \\
\text { ACCGAAGTGGCGGAGCTGGAACAGGAAGTGCAACGTCTG } \\
\text { GAGAACATTGTGAGCCAATACCGTACCCGTTATGGTCCGCT } \\
\text { G }\end{array}$ & $\begin{array}{l}\text { NTPIGKGPVLLPDN } \\
\text { HYLSTQSVLSKDPK } \\
\text { EKRDHMVLLEFVTA } \\
\text { AGITHGMDELYKLR } \\
\text { GSGSLEIEAAALEQE } \\
\text { NTALETEVAELEQE } \\
\text { VQRLENIVSQYRTR } \\
\text { YGPL }\end{array}$ \\
\hline $\mathrm{HCA}-Z_{\mathrm{E}}$ & $\begin{array}{l}\text { ATGAGCCACCACTGGGGTTACGGCAAACACAACGGTCCGG } \\
\text { AACACTGGCACAAAGACTTTCCGATTGCGAAGGGTGAACG } \\
\text { TCAGAGCCCGGTTGACATTGATACCCACACCGCGAAATACG } \\
\text { ACCCGAGCCTGAAGCCGCTGAGCGTGAGCTATGATCAGGC } \\
\text { GACCAGCCTGCGTATCCTGAACAACGGTCACGCGTTCAACG } \\
\text { TTGAATTTGACGATAGCCAAGACAAGGCGGTGCTGAAAGG } \\
\text { TGGCCCGCTGGATGGTACCTATCGTCTGATTCAGTTCCACTT } \\
\text { TCACTGGGGCAGCCTGGACGGTCAAGGCAGCGAACACACC } \\
\text { GTTGATAAGAAAAATACGCGGCGGAGCTGCACCTGGTGC } \\
\text { ACTGGAACACCAAATATGGTGACTTCGGCAAGGCGGTTCA } \\
\text { GCAACCGGATGGTCTGGCGGTGCTGGGCATCTTTCTGAAG } \\
\text { GTTGGTAGCGCGAAACCGGGCCTGCAGAAGGTGGTTGACG } \\
\text { TGCTGGATAGCATTAAAACCAAGGGTAAAAGCGCGGACTT } \\
\text { CACCAACTTTGATCCGCGTGGCCTGCTGCCGGAAAGCCTGG } \\
\text { ACTACTGGACCTACCCGGGTAGCCTGACCACCCCGCCGCTG } \\
\text { CTGGAGTGCGTGACCTGGATCGTTCTGAAAGAACCGATTA } \\
\text { GCGTGAGCAGCGAGCAAGTTCTGAAGTTCCGTAAACTGAA } \\
\text { CTTTAACGGCGAGGGCGAACCGGAGGAACTGATGGTTGAT } \\
\text { AACTGGCGTCCGGCGCAGCCGCTGAAGAACCGTCAAATCA } \\
\text { AGGCGAGCTTTAAACATATGGGTAGCGGCAGCCTGGAGAT } \\
\text { TGAAGCGGCGGCGCTGGAGCAGGAAAACACCGCGCTGGA } \\
\text { AACCGAGGTGGCGGAGCTGGAACAGGAAGTGCAACGTCT } \\
\text { GGAGAACATCGTGAGCCAATACCGTACCCGTTATGGCCCGC } \\
\text { TGCATCACCATCACCATCACTAA }\end{array}$ & $\begin{array}{l}\text { MSHHWGYGKHNG } \\
\text { PEHWHKDFPIAKG } \\
\text { ERQSPVDIDTHTAK } \\
\text { YDPSLKPLSVSYDQ } \\
\text { ATSLRILNNGHAFN } \\
\text { VEFDDSQDKAVLK } \\
\text { GGPLDGTYRLIQFH } \\
\text { FHWGSLDGQGSEH } \\
\text { TVDKKKYAAELHLV } \\
\text { HWNTKYGDFGKAV } \\
\text { QQPDGLAVLGIFLK } \\
\text { VGSAKPGLQKVVD } \\
\text { VLDSIKTKGKSADFT } \\
\text { NFDPRGLLPESLDY } \\
\text { WTYPGSLTTPPLLE } \\
\text { CVTWIVLKEPISVSS } \\
\text { EQVLKFRKLNFNGE } \\
\text { GEPEELMVDNWRP } \\
\text { AQPLKNRQIKASFK } \\
\text { HMGSGSLEIEAAAL } \\
\text { EQENTALETEVAEL } \\
\text { EQEVQRLENIVSQY } \\
\text { RTRYGPLHHHHHH }\end{array}$ \\
\hline$H G K-Z_{E}$ & $\begin{array}{l}\text { ATGCTGGACGATCGTGCGCGTATGGAAGCGGCGAAGAAA } \\
\text { GAAAAGGTGGAGCAGATCCTGGCGGAGTTTCAGCTGCAAG } \\
\text { AGGAAGACCTGAAGAAAGTTATGCGTCGTATGCAAAAGGA } \\
\text { GATGGATCGTGGTCTGCGTCTGGAAACCCACGAGGAAGCG } \\
\text { AGCGTGAAAATGCTGCCGACCTACGTTCGTAGCACCCCGGA } \\
\text { AGGTAGCGAGGTTGGCGACTTTCTGAGCCTGGATCTGGGT } \\
\text { GGCACCAACTTCCGTGTGATGCTGGTGAAAGTTGGTGAAG } \\
\text { GCGAGGAAGGTCAATGGAGCGTTAAGACCAAACACCAAAT } \\
\text { GTACAGCATTCCGGAGGATGCGATGACCGGTACCGCGGAA } \\
\text { ATGCTGTTTGATTATATCAGCGAGTGCATTAGCGACTTCCTG } \\
\text { GATAAGCACCAGATGAAACACAAGAAACTGCCGCTGGGTT }\end{array}$ & $\begin{array}{l}\text { MLDDRARMEAAK } \\
\text { KEKVEQILAEFQLQ } \\
\text { EEDLKKVMRRMQK } \\
\text { EMDRGLRLETHEEA } \\
\text { SVKMLPTYVRSTPE } \\
\text { GSEVGDFLSLDLGG } \\
\text { TNFRVMLVKVGEG } \\
\text { EEGQWSVKTKHQ } \\
\text { MYSIPEDAMTGTA } \\
\text { EMLFDYISECISDFL } \\
\text { DKHQMKHKKLPLG }\end{array}$ \\
\hline
\end{tabular}




\begin{tabular}{|c|c|c|}
\hline & $\begin{array}{l}\text { TCACCTTTAGCTTCCCGGTGCGTCACGAGGACATCGATAAG } \\
\text { GGTATTCTGCTGAACTGGACCAAGGGCTTTAAAGCGAGCG } \\
\text { GCGCGGAGGGTAACAACGTGGTTGGTCTGCTGCGTGACGC } \\
\text { GATTAAACGTCGTGGCGACTTCGAGATGGATGTGGTTGCG } \\
\text { ATGGTGAACGACACCGTTGCGACCATGATCAGCTGCTACTA } \\
\text { TGAGGATCACCAGTGCGAAGTGGGTATGATTGTTGGTACC } \\
\text { GGCTGCAACGCGTGCTACATGGAGGAAATGCAAAACGTGG } \\
\text { AACTGGTTGAGGGCGATGAAGGTCGTATGTGCGTGAACAC } \\
\text { CGAATGGGGCGCGTTTGGTGACAGCGGTGAACTGGATGAA } \\
\text { TTCCTGCTGGAGTATGACCGTCTGGTTGATGAAAGCAGCGC } \\
\text { GAACCCGGGTCAGCAACTGTACGAAAAGCTGATCGGTGGC } \\
\text { AAATATATGGGCGAGCTGGTGCGTCTGGTTCTGCTGCGTCT } \\
\text { GGTTGACGAGAACCTGCTGTTTCACGGTGAAGCGAGCGAA } \\
\text { CAGCTGCGTACCCGTGGCGCGTTTGAAACCCGTTTCGTGAG } \\
\text { CCAGGTTGAGAGCGACACCGGTGATCGTAAGCAAATCTAC } \\
\text { AACATTCTGAGCACCCTGGGCCTGCGTCCGAGCACCACCGA } \\
\text { CTGCGATATTGTGCGTCGTGCGTGCGAAAGCGTTAGCACCC } \\
\text { GTGCGGCGCACATGTGCAGCGCGGGTCTGGCGGGCGTGAT } \\
\text { CAACCGTATGCGTGAGAGCCGTAGCGAAGACGTTATGCGT } \\
\text { ATTACCGTGGGTGTTGATGGCAGCGTGTATAAGCTGCACCC } \\
\text { GAGCTTTAAAGAGCGTTTCCACGCGAGCGTTCGTCGTCTGA } \\
\text { CCCCGAGCTGCGAGATCACCTTCATTGAAAGCGAGGAAGG } \\
\text { TAGCGGTCGTGGTGCGGCGCTGGTGAGCGCGGTTGCGTGC } \\
\text { AAGAAAGCGTGCATGCTGGGTCAGCATATGGGCAGCGGTA } \\
\text { GCCTGGAGATTGAAGCGGCGGCGCTGGAGCAAGAAACA } \\
\text { CCGCGCTGGAAACCGAGGTGGCGGAGCTGGAACAGGAAG } \\
\text { TGCAACGTCTGGAGAACATTGTGAGCCAATACCGTACCCGT } \\
\text { TATGGCCCGCTGCATCACCATCACCATCACTAA }\end{array}$ & $\begin{array}{l}\text { FTFSFPVRHEDIDKG } \\
\text { ILLNWTKGFKASGA } \\
\text { EGNNVVGLLRDAIK } \\
\text { RRGDFEMDVVAM } \\
\text { VNDTVATMISCYYE } \\
\text { DHQCEVGMIVGTG } \\
\text { CNACYMEEMQNV } \\
\text { ELVEGDEGRMCVN } \\
\text { TEWGAFGDSGELD } \\
\text { EFLLEYDRLVDESSA } \\
\text { NPGQQLYEKLIGGK } \\
\text { YMGELVRLVLLRLV } \\
\text { DENLLFHGEASEQL } \\
\text { RTRGAFETRFVSQV } \\
\text { ESDTGDRKQIYNILS } \\
\text { TLGLRPSTTDCDIVR } \\
\text { RACESVSTRAAHM } \\
\text { CSAGLAGVINRMR } \\
\text { ESRSEDVMRITVGV } \\
\text { DGSVYKLHPSFKER } \\
\text { FHASVRRLTPSCEIT } \\
\text { FIESEEGSGRGAALV } \\
\text { SAVACKKACMLGQ } \\
\text { HMGSGSLEIEAAAL } \\
\text { EQENTALETEVAEL } \\
\text { EQEVQRLENIVSQY } \\
\text { RTRYGPLHHHHHH }\end{array}$ \\
\hline MSG-ZE & $\begin{array}{l}\text { ATGAGCCAGACCATCACCCAAAGCCGTCTGCGTATTGACGC } \\
\text { GAACTTCAAGCGTTTTGTGGATGAGGAAGTTCTGCCGGGTA } \\
\text { CCGGCCTGGATGCGGCGGCGTTCTGGCGTAACTTTGACGA } \\
\text { AATCGTTCACGATCTGGCGCCGGAGAACCGTCAGCTGCTG } \\
\text { GCGGAACGTGACCGTATTCAAGCGGCGCTGGATGAGTGGC } \\
\text { ACCGTAGCAACCCGGGCCCGGTTAAGGACAAAGCGGCGTA } \\
\text { CAAAAGCTTCCTGCGTGAACTGGGTTATCTGGTGCCGCAGC } \\
\text { CGGAGCGTGTGACCGTTGAAACCACCGGCATCGATAGCGA } \\
\text { GATTACCAGCCAAGCGGGTCCGCAACTGGTGGTTCCGGCG } \\
\text { ATGAACGCGCGTTATGCGCTGAACGCGGCGAACGCGCGTT } \\
\text { GGGGCAGCCTGTATGATGCGCTGTATGGTAGCGATATCATT } \\
\text { CCGCAGGAAGGTGCGATGGTTAGCGGTTACGACCCGCAGC } \\
\text { GTGGCGAACAAGTGATCGCGTGGGTTCGTCGTTTCCTGGAT } \\
\text { GAGAGCCTGCCGCTGGAAAACGGTAGCTATCAGGACGTGG } \\
\text { TTGCGTTTAAGGTGGTTGATAAACAGCTGCGTATCCAACTG } \\
\text { AAGAACGGCAAAGAAACCACCCTGCGTACCCCGGCGCAGT }\end{array}$ & $\begin{array}{l}\text { MSQTITQSRLRIDA } \\
\text { NFKRFVDEEVLPGT } \\
\text { GLDAAAFWRNFDE } \\
\text { IVHDLAPENRQLLA } \\
\text { ERDRIQAALDEWH } \\
\text { RSNPGPVKDKAAYK } \\
\text { SFLRELGYLVPQPER } \\
\text { VTVETTGIDSEITSQ } \\
\text { AGPQLVVPAMNAR } \\
\text { YALNAANARWGSL } \\
\text { YDALYGSDIIPQEGA } \\
\text { MVSGYDPQRGEQ } \\
\text { VIAWVRRFLDESLP } \\
\text { LENGSYQDVVAFKV } \\
\text { VDKQLRIQLKNGKE } \\
\text { TTLRTPAOFVGYRG }\end{array}$ \\
\hline
\end{tabular}




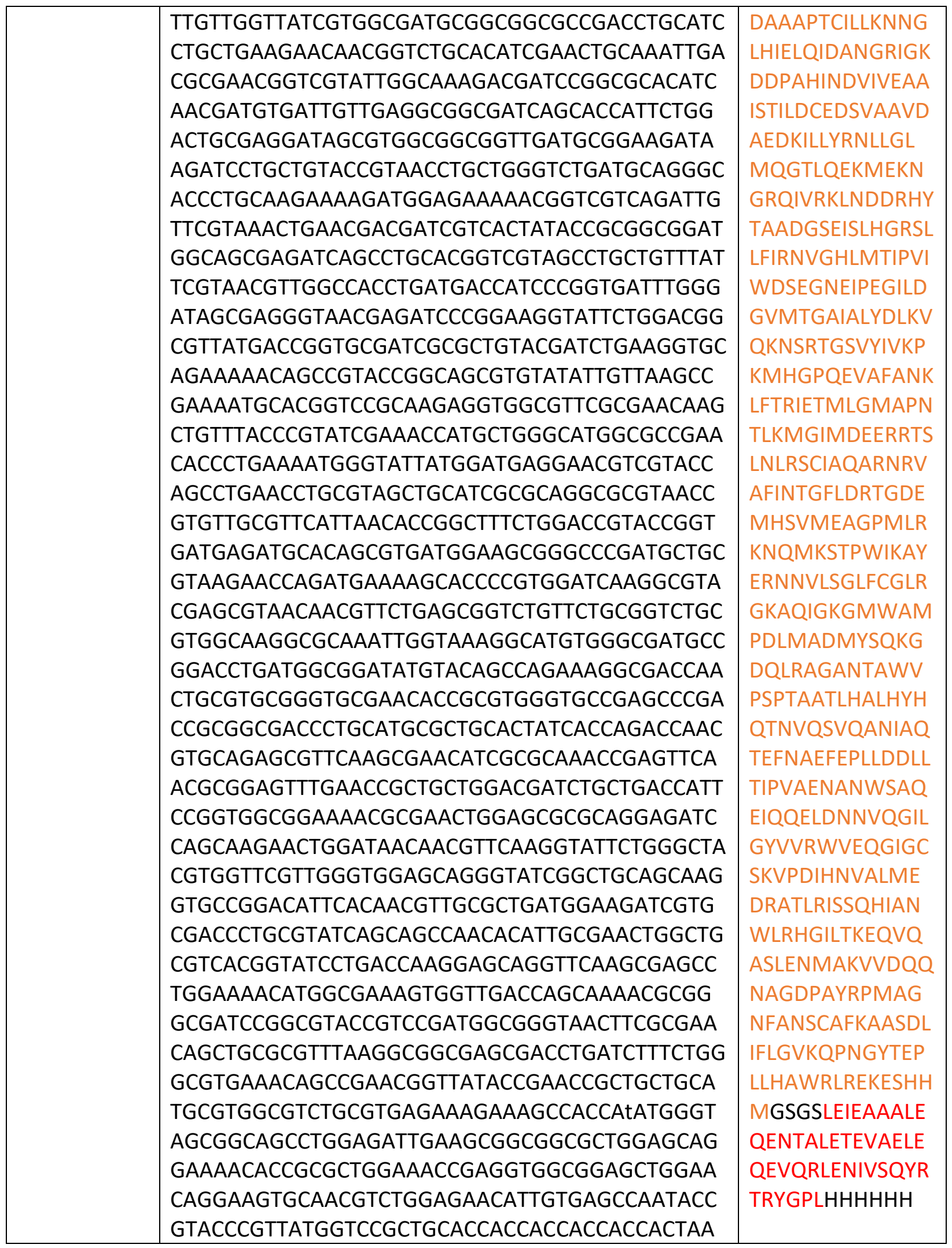

